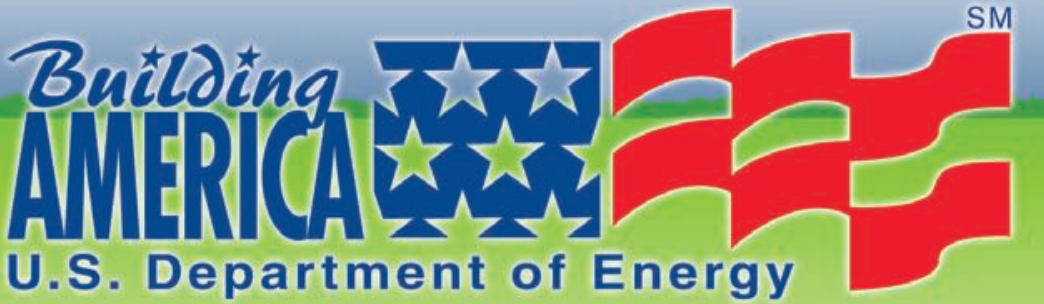

Research Toward Zero Energy Homes

\section{Research that Works}

May 2006 • NREL/TP-550-38238

\title{
Building America Performance Analysis Procedures for Existing Homes
}

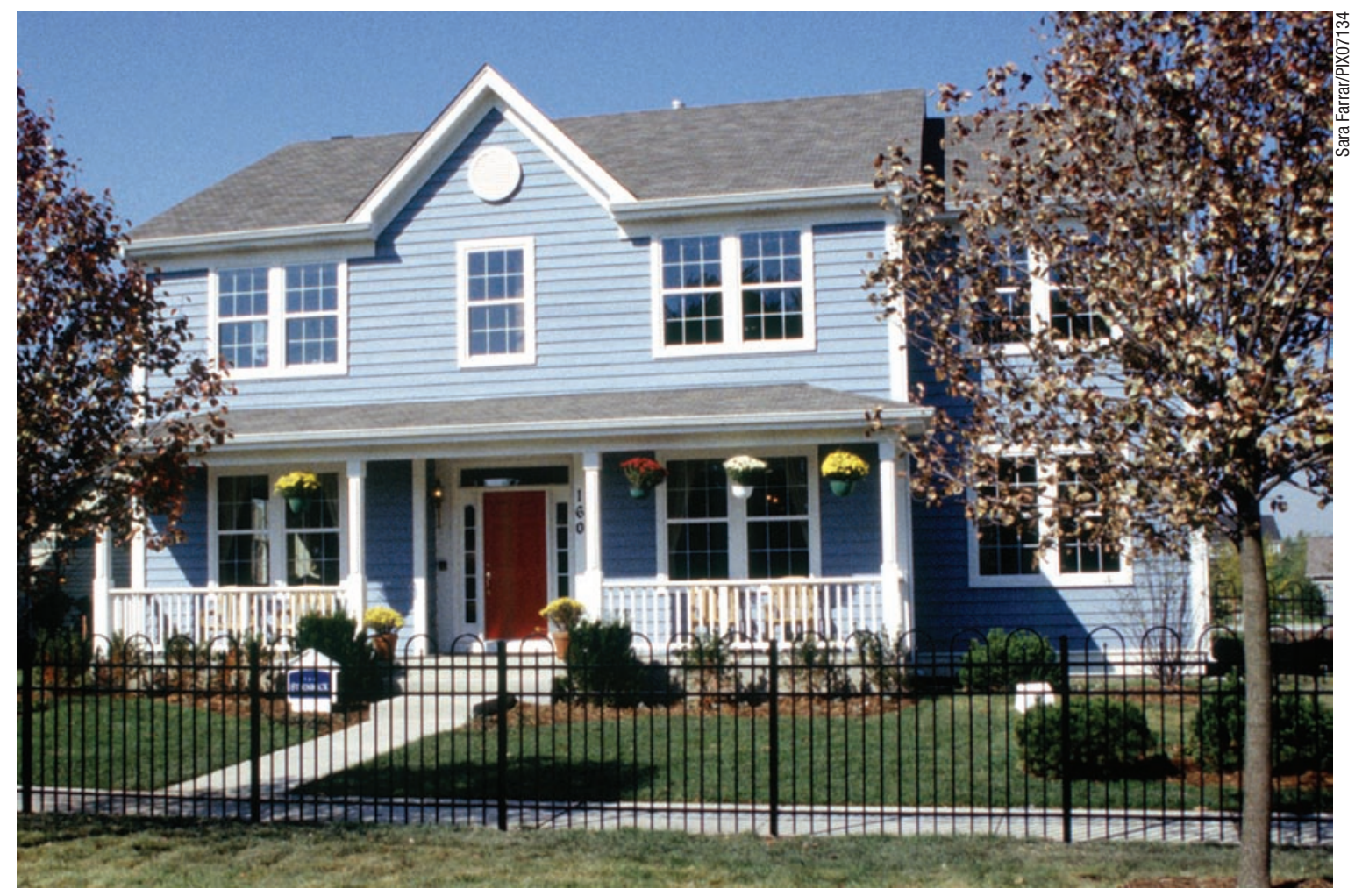

Robert Hendron

National Renewable Energy Laboratory 


\section{Building America Performance Analysis Procedures for Existing Homes}

R. Hendron

Prepared under Task No. BET6.8004
Technical Report NREL/TP-550-38238

May 2006

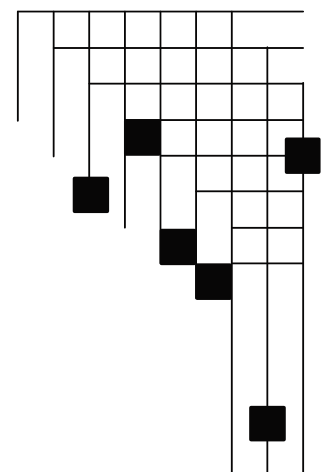




\section{NOTICE}

This report was prepared as an account of work sponsored by an agency of the United States government. Neither the United States government nor any agency thereof, nor any of their employees, makes any warranty, express or implied, or assumes any legal liability or responsibility for the accuracy, completeness, or usefulness of any information, apparatus, product, or process disclosed, or represents that its use would not infringe privately owned rights. Reference herein to any specific commercial product, process, or service by trade name, trademark, manufacturer, or otherwise does not necessarily constitute or imply its endorsement, recommendation, or favoring by the United States government or any agency thereof. The views and opinions of authors expressed herein do not necessarily state or reflect those of the United States government or any agency thereof.

Available electronically at http://www.osti.gov/bridge

Available for a processing fee to U.S. Department of Energy and its contractors, in paper, from:

U.S. Department of Energy

Office of Scientific and Technical Information

P.O. Box 62

Oak Ridge, TN 37831-0062

phone: 865.576 .8401

fax: 865.576 .5728

email: mailto:reports@adonis.osti.gov

Available for sale to the public, in paper, from:

U.S. Department of Commerce

National Technical Information Service

5285 Port Royal Road

Springfield, VA 22161

phone: 800.553 .6847

fax: 703.605.6900

email: orders@ntis.fedworld.gov

online ordering: http://www.ntis.gov/ordering.htm 


\section{Table of Contents}

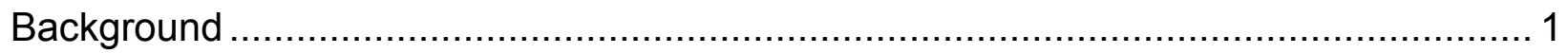

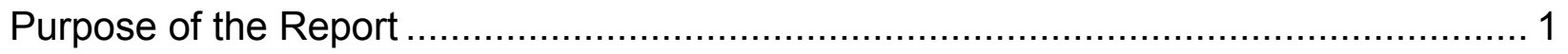

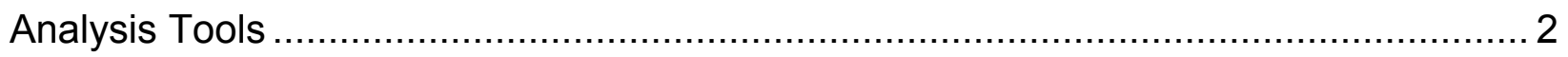

Modeling of the Pre-Retrofit Case ....................................................................... 3

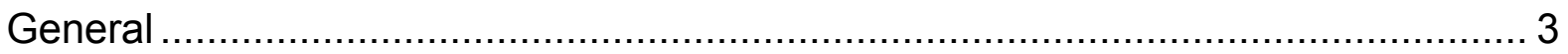

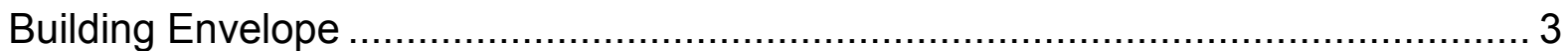

Space-Conditioning / Air-Distribution Equipment ................................................. 6

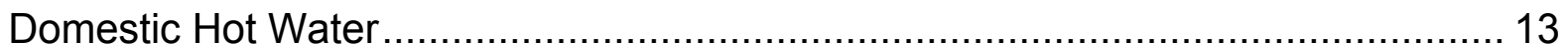

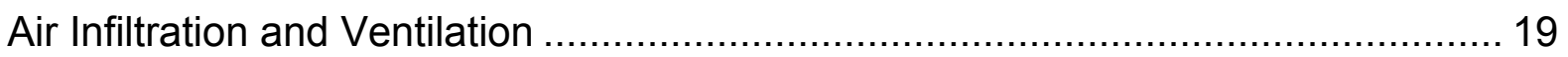

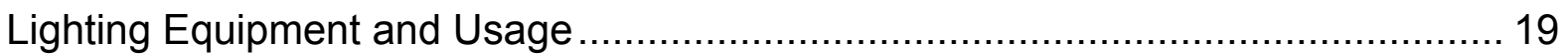

Appliances and Miscellaneous Electric Loads …............................................. 21

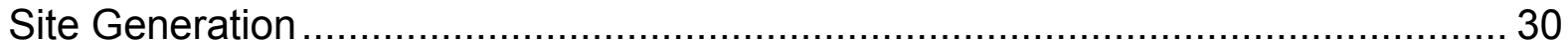

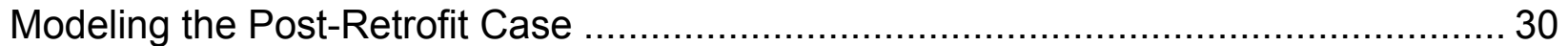

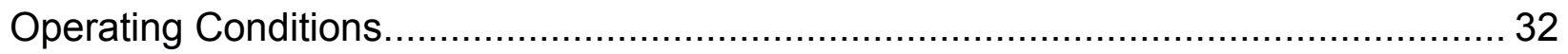

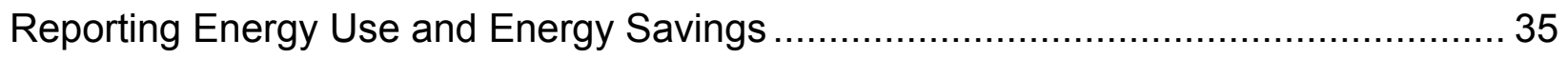

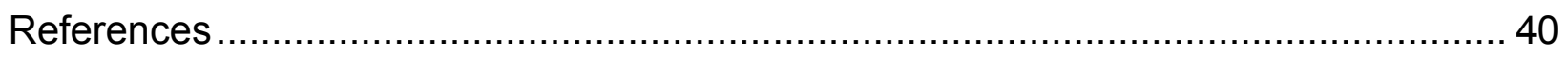




\section{List of Figures}

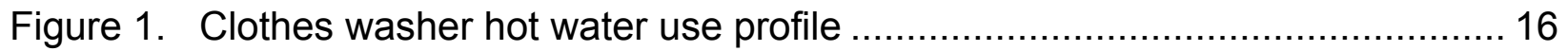

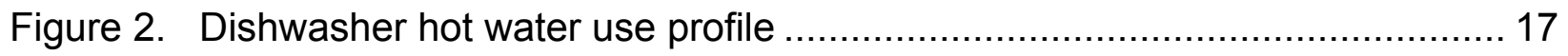

Figure 3. Shower, bath, and sink hot water use profile........................................ 17

Figure 4. Combined domestic hot water use profile ............................................ 18

Figure 5. Annual average interior lighting profile (built up from detailed profiles) ........ 21

Figure 6. Example of a detailed lighting profile (expressed as fraction of

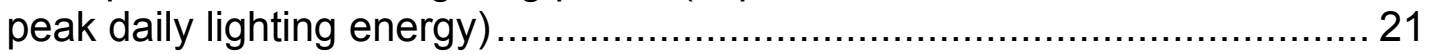

Figure 7. Total combined residential equipment profile …................................... 26

Figure 8. Refrigerator and freezer normalized energy use profile............................ 27

Figure 9. Clothes washer normalized machine energy use profile ........................... 27

Figure 10. Clothes dryer normalized energy use profile ......................................... 28

Figure 11. Dishwasher normalized energy use profile .............................................. 28

Figure 12. Range / oven normalized energy use profile ......................................... 29

Figure 13. Miscellaneous electric load normalized energy use profile ......................... 29

Figure 14. Average hourly load profile from occupants for all day-types and family types (16.5 hours/day/person total) ...................................... 34

Figure 15. Detailed hourly load profiles resulting from occupants being in different parts of the house on weekdays (WD) and weekends (WE) ......... 34 


\section{List of Tables}

Table 1. Default R-Values for Wall Cavity Insulation ............................................. 4

Table 2. Default R-Values for Floors above Unconditioned Space.............................. 4

Table 3. Default R-Values for Common Insulation Types ........................................... 4

Table 4. Default Solar Adsorptances for Common Roofing Surfaces ........................... 5

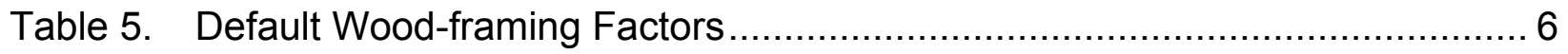

Table 6. Default Furnace and Boiler System Efficiencies, "Gas" refers to

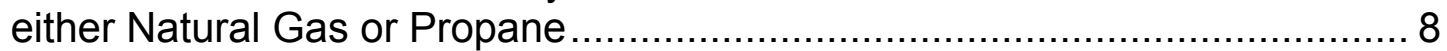

Table 7. Default Heating-System Blower and Auxiliary Electricity Consumption .......... 9

Table 8. Default Air Conditioning and Heat-Pump Efficiencies................................. 10

Table 9. Default DHW Energy Factors, Known Equipment Characteristics. "Gas" Refers to Either Natural Gas or Propane ....................................... 14

Table 10. Default Domestic Hot Water Consumption by End Use .............................. 15

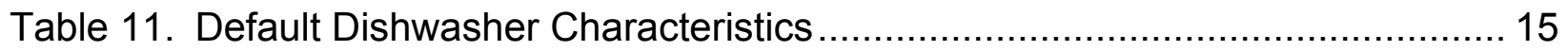

Table 12. Default Standard Size $\left(\sim 2.5 \mathrm{ft}^{3}\right)$ Clothes Washer Characteristics ................. 16

Table 13. Default Lighting Operating Hours for Common Room Types....................... 20

Table 14. Default Gas Clothes Dryer Characteristics (Assumes Typical 1990 Clothes Washer Capacity and Remaining Moisture Content) ...................... 22

Table 15. Default Electric Clothes Dryer Characteristics (Assumes Typical 1990 Clothes Washer Capacity and Remaining Moisture Content)............. 23

Table 16. Default Gas Over / Cooktop Characteristics .............................................. 23

Table 17. Default Electric Oven / Cooktop Characteristics ........................................... 24

Table 18. Default Internal Loads from Appliances and Small Electric End-Uses in the Pre-Retrofit Case .................................................. 25

Table 19. Peak Sensible and Latent Heat Gain from Occupants ............................... 33 
Table 20. Example Summary of Site Energy Consumption by End-Use for an Existing Homes Project............................................................... 36

Table 21. Example Summary of Source Energy Consumption by End-Use for an Existing Homes Project ........................................................... 37

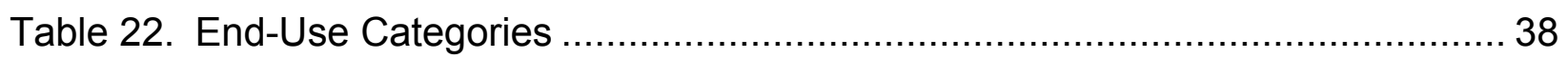

Table 23. Example Measure Savings Report for an Existing Homes Project ............... 39 


\section{Definitions}

$\mathrm{ACH}$

AFUE

ASHRAE

ASTM

BA

BESTEST

CAAfue

CFA

DOE

DOE-2

DHW

DSE

DUF

ECM

EER

EF

EIA

ELCAP

EPA

EPRI

EPS

FFA

$\mathrm{H}$

HERS

HSPF

LBNL

$\mathrm{M}$

MAT air changes per hour

Annual Fuel Utilization Efficiency

American Society of Heating, Refrigerating and AirConditioning Engineers

American Society for Testing and Materials

Building America

A benchmark for building energy simulation: Building Energy Simulation Test and Diagnostic Method

combined appliance AFUE

conditioned floor area

U.S. Department of Energy

building energy analysis program that can predict the energy use and cost for all types of buildings

domestic hot water

distribution system efficiency

dryer usage factor

electronically commutated motor

energy efficiency ratino

efficiency factor

Energy Information Administration

End-Use Load and Consumer Assessment Program

U.S. Environmental Protection Agency

Electric Power Research Institute

expanded polystyrene

finished floor area

height of one story

Home Energy Rating System developed by RESNET

heating seasonal performance factor

Lawrence Berkeley National Laboratory

maintenance factor

monthly average temperatures 
MEF

MEL

NAECA

NREL

$\mathrm{OA}$

RECS

RESNET

SDT

SEER

SHGC

TMY2

TREAT

TRNSYS

TXV

U-values

UA

WDT

XPS modified energy factor

miscellaneous electric loads

National Appliance Energy Conservation Act

National Renewable Energy Laboratory

outdoor air

EIA Residential Energy Consumption Survey

Residential Energy Service Network

summer design temperatures

seasonal energy efficiency ratio

solar heat gain coefficient

Typical Meteorological Year weather data (1961-90)

Building energy software that performs hourly simulations for single-family, multi-family, and mobile homes

The Transient Energy System Simulation Tool is software designed to simulate the transient performance of thermal energy systems

thermostatic expansion valve

The thermal transmittance of a material, incorporating the thermal conductance of the structure along with heat transfer resulting from convection and radiation

heat loss coefficient

winter design temperatures

extruded polystyrene 


\section{Building America Performance Analysis Procedures for Existing Homes}

\section{Background}

Because there are more than 101 million residential households in the United States today, it is not surprising that existing residential buildings represent an extremely large source of potential energy savings. Because thousands of these homes are renovated each year, the U.S. Department of Energy's (DOE's) Building America program is investigating the best ways to make existing homes more energy-efficient, based on lessons learned from research in new homes. The Building America research goals target a 20\% reduction in energy use in existing homes by 2015 and a $30 \%$ reduction by 2025 .

The strategy for the existing homes project of Building America is to establish technology pathways that reduce energy consumption cost-effectively in American homes. The existing buildings project focuses on finding ways to adapt the results from the new homes research to retrofit applications in existing homes. Research activities include a combination of computer modeling, field demonstrations, and long-term monitoring to support the development of integrated approaches to reduce energy use in existing residential buildings. Analytical tools are being developed to guide designers and builders in selecting the best approaches for each application. Also, DOE partners with the U.S. Environmental Protection Agency (EPA) to increase energy efficiency in existing homes through the Home Performance with ENERGY STAR ${ }^{\circledR}$ program (www.energystar.gov/index.cfm?c=home_improvement.hm_improvement_hpwes).

\section{Purpose of the Report}

This report provides a proposed set of guidelines for estimating the energy savings achieved by a package of retrofits or an extensive rehabilitation of an existing home. Building America has developed a set of typical operating conditions that will be used for the purpose of using a building simulation model to objectively compare energy use before and after a series of retrofits are completed. Actual occupant behavior is extremely important for the purpose of determining the cost-effectiveness of a retrofit package, especially if the homeowner is paying the bills. But for the purpose of tracking progress toward programmatic goals, and for comparing the performance of one house to another, it is essential to use a hypothetical set of occupants with typical behavioral patterns. These typical operating conditions are fully consistent with those used in the Building America Research Benchmark for new construction (which we will refer to simply as the Benchmark) (Hendron 2005).

Certain field test and audit methods are also described in this report. These tests help establish accurate building system performance characteristics that are needed for a meaningful simulation of whole-house energy use. Several sets of default efficiency values have also been developed for certain older appliances that cannot be easily tested and for which published specifications are not readily available. 


\section{Analysis Tools}

A key issue in any building energy analysis is which tool or program should be chosen to estimate energy consumption. An hourly simulation is often necessary to fully evaluate the timedependent energy impacts of advanced systems used in Building America houses. Thermal mass, solar heat gain, and wind-induced air infiltration are examples of time-dependent effects that can be accurately modeled only by using a model that calculates heat transfer and temperature in short time intervals. In addition, an hourly simulation program is necessary to accurately estimate peak energy loads. Because of the large number of users, public availability, and level of technical support, DOE-2 is the hourly simulation tool recommended for systems analysis studies performed under the DOE Building America program.

EnergyGauge $^{1}$ is a frequently used interface for DOE-2 because it has been tailored specifically to residential buildings. However, it was not designed with existing homes as the top priority, so retrofits must be analyzed by comparing separate runs. Team analysts are encouraged to use other simulation tools when appropriate for specialized building simulation analysis, provided the tool has met the requirements of BESTEST in accordance with the software certification sections of the RESNET/HERS Guidelines (RESNET 2002). Regardless of the tool selected, teams should present complete analysis results, in accordance with the reporting guidelines described later in this section.

Most of the simulation tools that are useful for new residential construction are also applicable to residential retrofit analysis. Certain tools, such as TREAT, offer additional features such as sideby-side comparisons, automated efficiency package recommendations, and utility bill analysis/reconciliation. Further information about TREAT and a number of other useful tools for retrofit analysis of both residential and commercial buildings can be found in the DOE Energy Software Tools Directory (www.eere.energy.gov/buildings/tools_directory).

NREL does not recommend that utility bills be heavily relied upon as a tool for model validation, except as an approximate check of model accuracy. There are two important reasons for this position:

- It is extremely difficult to accurately determine occupant behavior during the time period reflected in the utility bills.

- The large number of uncertain input parameters allows multiple ways to reconcile the model with the small number of utility bills, and no reliable methodology exists for performing this calibration because the problem is fundamentally mathematically underdetermined.

Instead, detailed inspections, short-term testing, and long-term monitoring should be utilized to the greatest extent possible to minimize the uncertainty in model inputs. Default values may be used when certain building features are inaccessible (wall insulation) or efficiency characteristics cannot be readily determined through inspection or short-term testing (furnace AFUE). The effects of maintenance and repairs should always be considered when using default values for equipment efficiency or the amount of insulation.

Throughout the remainder of this section, the term "Pre-Retrofit Case" refers to the state of an existing house immediately before it undergoes a series of upgrades, repairs, additions, or

\footnotetext{
${ }^{1}$ This is available for purchase from the Florida Solar Energy Center (http://energygauge.com/).
} 
renovations. These measures may be limited to a focused set of energy efficiency improvements to the house or may be part of a larger remodeling or gut rehabilitation effort. The terms "PostRetrofit Case" and "Prototype" refer to the same existing house after the package of improvements is complete.

\section{Modeling the Pre-Retrofit Case}

\section{General}

Any element of the Pre-Retrofit Case that is not specifically addressed in the following sections, or is not changed as part of the package of energy efficiency measures, is assumed to be the same as the Post-Retrofit Case.

\section{Building Envelope}

To the extent possible, all building envelope components (including walls, windows, foundation, roof, and floors) for the Pre-Retrofit Case shall be based on physical inspections, audits, design specifications, or measured data. Co-heating (Judkoff et al. 2000), or infrared imaging during cold weather can provide useful information about the insulation quality of the house without damaging the building envelope. A Short-Term Monitoring Tests (STEM) can provide the overall building loss coefficient and thermal mass (Judkoff et al. 2000), but in most retrofit scenarios a STEM test is overly expensive and would not provide data that could be easily factored into a building simulation.

If detailed envelope characteristics cannot be obtained, the following default specifications may be used:

- R-values for cavity insulation in exterior $2 \times 4$ or $2 \times 6$ wood frame walls from Table 1

- R-values for cavity insulation in floors over unconditioned space from Table 2

- Insulation thickness in all other locations shall be measured, and the default R-values per inch in Table 3 shall be applied

- Default U-values for vertical fenestration, including windows and sliding glass doors, from Table 4 in Chapter 31 of the 2005 ASHRAE Handbook of Fundamentals (ASHRAE 2005)

- Total assembly SHGC for vertical fenestration from Table 13 in Chapter 31 of the 2005 ASHRAE Handbook of Fundamentals

- Default solar absorptivity equal to 0.50 for opaque areas of exterior walls and from Table 4 for opaque areas of roofs

- Default total infrared emittance of exterior walls and roofs equal to 0.90

- The default framing factors in Table 5 may be used for houses using wood construction. 
Table 1. Default R-values for Wall Cavity Insulation (Based in Part on Huang \& Gu 2002)

\begin{tabular}{|c|c|c|c|c|}
\hline \multirow{2}{*}{$\begin{array}{c}\text { Wall Construction } \\
\text { Type }\end{array}$} & \multicolumn{4}{|c|}{ Year of Construction } \\
\cline { 2 - 5 } & $\mathbf{1 9 9 0 +}$ & $\mathbf{1 9 8 0 - 8 9}$ & $\mathbf{1 9 5 0 - 7 9}$ & Pre 1950 \\
\hline $2 \times 4,16$ in. o.c. & 13 & 11 & 9 & 7 \\
\hline $2 \times 6,24$ in. o.c. & 19 & 17 & 15 & 13 \\
\hline
\end{tabular}

Table 2. Default R-Values for Floors above Unconditioned Space (Based in Part on Huang \& Gu 2002)

\begin{tabular}{|c|c|c|c|c|}
\hline \multirow{2}{*}{$\begin{array}{c}\text { Building America } \\
\text { Climate Region }\end{array}$} & \multicolumn{4}{|c|}{ Year of Construction } \\
\cline { 2 - 5 } & $\mathbf{1 9 9 0 +}$ & $\mathbf{1 9 8 0 - 8 9}$ & $\mathbf{1 9 5 0 - 7 9}$ & Pre 1950 \\
\hline $\begin{array}{c}\text { Cold, Very Cold, } \\
\text { Subarctic, Marine }\end{array}$ & 19 & 17 & 15 & 13 \\
\hline All Others & 0 & 0 & 0 & 0 \\
\hline
\end{tabular}

Table 3. Default R-value for Common Insulation Types (DOE 2003, E-Star Colorado 2005)

\begin{tabular}{|c|c|c|}
\hline \multirow{2}{*}{ Insulation Material } & \multicolumn{2}{|c|}{ Year of Construction } \\
\hline & 1990 or after & Before 1990 \\
\hline High-Density Fiberglass Batt & $3.8 /$ in. & $3.0 /$ in. \\
\hline Low-Density Fiberglass Batt & $2.7 /$ in. & $2.0 /$ in. \\
\hline Loose-Fill Fiberglass & $3.2 /$ in. & $2.5 /$ in. \\
\hline Cellulose (Blown, Wet, or Dry) & $3.7 /$ in. & $3.4 /$ in. \\
\hline Expanded Polystyrene (EPS) & $4.0 /$ in. & $3.8 /$ in. \\
\hline Extruded Polystyrene (XPS) & $5.0 /$ in. & $4.8 /$ in. \\
\hline Open-Cell Polyurethane Foam & $3.6 /$ in. & $3.3 /$ in. \\
\hline Closed-Cell Polyurethane Foam & $6.5 /$ in. & $5.9 /$ in. \\
\hline Rigid Polyisocyanurate & $7.2 /$ in. & $5.8 /$ in. \\
\hline
\end{tabular}


Table 4. Default Solar Absorptances for Common Roofing Surfaces (Parker et al. 2000)

\begin{tabular}{|c|c|c|c|}
\hline Roof Material & Absorptance & Roof Material & Absorptance \\
\hline Composition Shingles & & Wood Shingles & \\
\hline Dark & 0.92 & Dark & 0.90 \\
\hline Medium & 0.85 & Medium & 0.80 \\
\hline \multirow[t]{2}{*}{ Light } & 0.75 & & \\
\hline & & Concrete/Cement & \\
\hline Tile/Slate & & Dark & 0.90 \\
\hline Dark & 0.90 & Medium & 0.75 \\
\hline Medium & 0.75 & Light & 0.60 \\
\hline Terra cotta & 0.65 & White & 0.30 \\
\hline Light & 0.60 & & \\
\hline \multirow[t]{2}{*}{ White } & 0.30 & Membrane & \\
\hline & & Dark & 0.90 \\
\hline Metal & & Medium & 0.75 \\
\hline Dark & 0.90 & Light & 0.60 \\
\hline Medium & 0.75 & White & 0.30 \\
\hline Galvanized, unfinished & 0.70 & & \\
\hline Light & 0.60 & Built-up (gravel surface) & \\
\hline Galvalum, unfinished & 0.35 & Dark & 0.92 \\
\hline \multirow[t]{2}{*}{ White } & 0.30 & Medium & 0.85 \\
\hline & & Light & 0.75 \\
\hline
\end{tabular}


Table 5. Default Wood-framing Factors

\begin{tabular}{|c|c|c|}
\hline $\begin{array}{c}\text { Enclosure } \\
\text { Element }\end{array}$ & $\begin{array}{c}\text { Frame Spacing } \\
\text { (in. o.c.) }\end{array}$ & $\begin{array}{c}\text { Framing } \\
\text { Fraction } \\
\text { (\% area) }\end{array}$ \\
\hline Walls & 16 & $23 \%$ \\
\hline Floors & 16 & $13 \%$ \\
\hline Ceilings & 24 & $11 \%$ \\
\hline
\end{tabular}

\section{Space Conditioning / Air-Distribution Equipment}

To the extent possible, the performance characteristics (efficiency and capacity) of all spaceconditioning components (including heating system, cooling system, dehumidification, air handler, and ducts) for the Pre-Retrofit Case shall be based on physical inspections, audits, design specifications, and measured data. An estimate of Annual Fuel Utilization Efficiency (AFUE) for a furnace or Heating Seasonal Performance Factor (HSPF) for a heat pump can be estimated by performing a co-heating test to determine the building loss coefficient (Judkoff et al. 2000), then measuring the gas or electricity input over a period of time with known inside and outside temperatures. Because thermal mass and solar effects complicate this approach, it should ideally be conducted under near steady-state conditions at night. Field-audit procedures for heating equipment have also been developed by LBNL (Szydlowski and Cleary 1988). Cooling efficiency is much more difficult to measure directly; in most cases, the manufacturers published data must be used — or default values if published performance data are not available.

Default furnace or boiler system efficiency may be calculated using Equation 1 in conjunction with the parameters in Table 6 if the actual efficiency of the equipment is unknown and cannot be readily obtained through field-testing (for example, if the audit is conducted in the summer, the heating system is broken, or testing would be cost-prohibitive). Typical base values for AFUE were obtained from the ASHRAE HVAC Systems and Equipment Handbook (ASHRAE 2004a), the 1987 EPRI Technical Assessment Guide (EPRI 1987), and the Technical Support Documents for the NAECA appliance standards (DOE 2004a). Default AFUE values for system configurations not listed in Table 6 may be estimated using these references. Estimates of degradation rates are partly based on the E-Source Space Heating Technology Atlas (E-Source 1993).

Equation 1: $\quad$ AFUE $=($ Base AFUE $) *(1-M)^{\text {age }}$

Where:

Base AFUE = Typical efficiency of Pre-Retrofit equipment when new

$\mathrm{M}=$ Maintenance Factor

Age $=$ Age of equipment in years 
For example, the default AFUE for a 10-year-old, poorly maintained, oil furnace with a conventional burner would be calculated as follows:

$$
\operatorname{AFUE}=(71) *(1-0.025)^{10}=55 \% .
$$

Auxiliary electricity use for furnaces and boilers, including blowers and controls, shall be measured directly if possible. If accurate measurements cannot be made, the default values of auxiliary electricity use in Table 7 may be used.

The default air-conditioner and heat-pump efficiencies in Table 8 may be used if the actual efficiency cannot be calculated or measured. Base values for SEER, EER, and HSPF were obtained from the engineering analysis of appliance standards for air conditioners and heat pumps (DOE 2002), and from the LBNL Energy Data Sourcebook (Wenzel et al. 1997). Default efficiencies for equipment not listed in the table may either be interpolated or estimated by referring to the original references. Adjustments to efficiency related to age and quality of maintenance shall be applied in accordance with Equation 2. Performance degradation rates for cooling systems are based in part on a study done by LBNL for the California Energy Commission (Matson et al. 2002).

\section{Equation 2: $\quad \mathbf{E F F}=($ Base EFF $) *(1-M)^{\text {age }}$}

Where:

Base EFF = Typical efficiency of Pre-Retrofit equipment when new (SEER, EER, or HSPF)

$\mathrm{M}=$ Maintenance Factor

Age $=$ Age of equipment in years. 
Table 6. Default Furnace and Boiler System Efficiencies;

"Gas" Refers to Either Natural Gas or Propane

\begin{tabular}{|l|c|c|c|}
\hline & Base & \multicolumn{2}{|c|}{ Maintenance Factor (M) } \\
\cline { 3 - 4 } Type of Space-Heating Equipment & AFUE* & $\begin{array}{c}\text { Annual } \\
\text { Professional } \\
\text { Maintenance }\end{array}$ & $\begin{array}{c}\text { Seldom or } \\
\text { Never } \\
\text { Maintained }\end{array}$ \\
\hline Condensing gas furnace & 90 & 0.005 & 0.015 \\
\hline $\begin{array}{l}\text { Gas furnace, direct-vent or forced- } \\
\text { draft combustion, electronic ignition, } \\
\text { in conditioned space }\end{array}$ & 80 & 0.005 & 0.015 \\
\hline $\begin{array}{l}\text { Gas furnace, natural-draft } \\
\text { combustion, vent damper, electronic } \\
\text { ignition, in conditioned space }\end{array}$ & 78 & 0.005 & 0.015 \\
\hline $\begin{array}{l}\text { Gas furnace, natural-draft } \\
\text { combustion, standing pilot light, in } \\
\text { conditioned space }\end{array}$ & 75 & 0.005 & 0.015 \\
\hline $\begin{array}{l}\text { Gas furnace, natural-draft } \\
\text { combustion, standing pilot light, no } \\
\text { vent damper, in unconditioned space }\end{array}$ & 64 & 0.005 & 0.015 \\
\hline $\begin{array}{l}\text { Gas hot water boiler, natural-draft } \\
\text { combustion, standing pilot light }\end{array}$ & 80 & 0.005 & 0.015 \\
\hline Gas steam boiler & 81 & 0.005 & 0.015 \\
\hline Condensing gas boiler & 90 & 0.005 & 0.015 \\
\hline $\begin{array}{l}\text { Gas hot water / fan-coil combo } \\
\text { system }\end{array}$ & 80 & 0.005 & 0.015 \\
\hline $\begin{array}{l}\text { Gas boiler / tankless coil combo } \\
\text { system }\end{array}$ & 80 & 0.005 & 0.015 \\
\hline Gas space heater, fan type & 0.005 & 0.015 \\
\hline Gas space heater, gravity type & & 0.01 & 0.025 \\
\hline $\begin{array}{l}\text { Oil furnace, flame-retention burner, } \\
\text { vent dampers, in conditioned space }\end{array}$ & 81 & & 0.015 \\
\hline
\end{tabular}


Table 6 continued. Default Furnace and Boiler System Efficiencies;

"Gas" Refers to Either Natural Gas or Propane

\begin{tabular}{|c|c|c|c|}
\hline \multirow[b]{2}{*}{ Type of Space-Heating Equipment } & \multirow{2}{*}{$\begin{array}{c}\text { Base } \\
\text { AFUE* }\end{array}$} & \multicolumn{2}{|c|}{ Maintenance Factor (M) } \\
\hline & & $\begin{array}{c}\text { Annual } \\
\text { Professional } \\
\text { Maintenance }\end{array}$ & $\begin{array}{c}\text { Seldom or } \\
\text { Never } \\
\text { Maintained }\end{array}$ \\
\hline $\begin{array}{l}\text { Oil furnace, conventional burner, no } \\
\text { vent dampers, in conditioned space }\end{array}$ & 71 & 0.01 & 0.025 \\
\hline $\begin{array}{l}\text { Oil hot water boiler, forced-draft } \\
\text { combustion }\end{array}$ & 80 & 0.01 & 0.025 \\
\hline Oil steam boiler & 82 & 0.01 & 0.025 \\
\hline $\begin{array}{l}\text { Electric-resistance furnace or boiler, } \\
\text { conditioned space }\end{array}$ & 100 & 0 & 0 \\
\hline $\begin{array}{l}\text { Electric-resistance furnace or boiler, } \\
\text { unconditioned space }\end{array}$ & 98 & 0.001 & 0.001 \\
\hline $\begin{array}{l}\text { Electric-resistance baseboard } \\
\text { heating }\end{array}$ & 100 & 0 & 0 \\
\hline Electric space heater & 100 & 0 & 0 \\
\hline
\end{tabular}

* Combined Appliance AFUE ( $\left.\mathrm{CA}_{\mathrm{AFUE}}\right)$ for combo systems

Table 7. Default Heating-System Blower and Auxiliary Electricity Consumption

\begin{tabular}{|l|c|}
\hline \multicolumn{1}{|c|}{ Type of Heating Equipment } & Electricity/Capacity \\
\hline Gas furnace (including mobile-home furnace) & $9.2(\mathrm{kWh} / \mathrm{yr}) /(\mathrm{kBtu} / \mathrm{hr})$ \\
\hline Gas hot water boiler with hydronic distribution & $1.1(\mathrm{kWh} / \mathrm{yr}) /(\mathrm{kBtu} / \mathrm{hr})$ \\
\hline Gas boiler with forced-air distribution & $9.2(\mathrm{kWh} / \mathrm{yr}) /(\mathrm{kBtu} / \mathrm{hr})$ \\
\hline Oil furnace & $8.0(\mathrm{kWh} / \mathrm{yr}) /(\mathrm{kBtu} / \mathrm{hr})$ \\
\hline Oil hot water boiler with hydronic distribution & $2.3(\mathrm{kWh} / \mathrm{yr}) /(\mathrm{kBtu} / \mathrm{hr})$ \\
\hline Electric furnace & Included in AFUE \\
\hline
\end{tabular}


Table 8. Default Air-Conditioning and Heat-Pump Efficiencies

\begin{tabular}{|c|c|c|c|c|c|}
\hline \multirow{2}{*}{$\begin{array}{l}\text { Type of Air-Conditioning or } \\
\text { Heat-Pump Equipment }\end{array}$} & \multirow{2}{*}{$\begin{array}{l}\text { Base } \\
\text { SEER }\end{array}$} & \multirow{2}{*}{$\begin{array}{l}\text { Base } \\
\text { EER }\end{array}$} & \multirow{2}{*}{$\begin{array}{l}\text { Base } \\
\text { HSPF }\end{array}$} & \multicolumn{2}{|c|}{ Maintenance Factor (M) } \\
\hline & & & & $\begin{array}{l}\text { Annual } \\
\text { Professional } \\
\text { Maintenance }\end{array}$ & $\begin{array}{l}\text { Seldom or } \\
\text { Never } \\
\text { Maintained }\end{array}$ \\
\hline $\begin{array}{l}\text { Split central air conditioner, two- } \\
\text { speed reciprocating compressor, } \\
\text { electronically commutated air } \\
\text { handler motor (ECM), } \\
\text { thermostatic expansion valve } \\
\text { (TXV), fan coil }\end{array}$ & 14 & 10.5 & & 0.01 & 0.02 \\
\hline $\begin{array}{l}\text { Split central air conditioner, } \\
\text { single-speed scroll compressor, } \\
\text { ECM air handler motor, cased } \\
\text { coil }\end{array}$ & 12 & 10.8 & & 0.01 & 0.03 \\
\hline $\begin{array}{l}\text { Split central air conditioner, } \\
\text { single-speed reciprocating } \\
\text { compressor, PSC air-handler } \\
\text { motor, cased coil (after 1991) }\end{array}$ & 10 & 9.3 & & 0.01 & 0.03 \\
\hline $\begin{array}{l}\text { Split central air conditioner, } \\
\text { single-speed reciprocating } \\
\text { compressor, PSC air-handler } \\
\text { motor, cased coil (1981-1991) }\end{array}$ & 8 & 7.7 & & 0.01 & 0.03 \\
\hline $\begin{array}{l}\text { Split central air conditioner, } \\
\text { single-speed reciprocating } \\
\text { compressor, PSC air-handler } \\
\text { motor, cased coil (before 1981) }\end{array}$ & 6.5 & 6.4 & & 0.01 & 0.03 \\
\hline $\begin{array}{l}\text { Split heat pump, single-speed } \\
\text { scroll compressor, ECM air } \\
\text { handler motor, TXV valve }\end{array}$ & 14 & 10.5 & 8.0 & 0.01 & 0.03 \\
\hline
\end{tabular}


Table 8 continued. Default Air Conditioning and Heat-Pump Efficiencies

\begin{tabular}{|c|c|c|c|c|c|}
\hline \multirow{2}{*}{$\begin{array}{l}\text { Type of Air-Conditioning or } \\
\text { Heat-Pump Equipment }\end{array}$} & \multirow{2}{*}{$\begin{array}{l}\text { Base } \\
\text { SEER }\end{array}$} & \multirow{2}{*}{$\begin{array}{l}\text { Base } \\
\text { EER }\end{array}$} & \multirow{2}{*}{$\begin{array}{l}\text { Base } \\
\text { HSPF }\end{array}$} & \multicolumn{2}{|c|}{ Maintenance Factor (M) } \\
\hline & & & & $\begin{array}{l}\text { Annual } \\
\text { Professional } \\
\text { Maintenance }\end{array}$ & $\begin{array}{l}\text { Seldom or } \\
\text { Never } \\
\text { Maintained }\end{array}$ \\
\hline $\begin{array}{l}\text { Split heat pump, single-speed } \\
\text { reciprocating compressor, PSC } \\
\text { air-handler motor (after 1991) }\end{array}$ & 10 & 9.3 & 7.1 & 0.01 & 0.03 \\
\hline $\begin{array}{l}\text { Split heat pump, single-speed } \\
\text { reciprocating compressor, PSC } \\
\text { air-handler motor (1981-1991) }\end{array}$ & 8 & 7.7 & 6.6 & 0.01 & 0.03 \\
\hline $\begin{array}{l}\text { Split heat pump, single-speed } \\
\text { reciprocating compressor, PSC } \\
\text { air-handler motor (before 1981) }\end{array}$ & 6.5 & 6.4 & 6.0 & 0.01 & 0.03 \\
\hline $\begin{array}{l}\text { Packaged central air conditioner, } \\
\text { single-speed reciprocating } \\
\text { compressor, PSC air-handler } \\
\text { motor }\end{array}$ & 10 & 9.1 & & 0.01 & 0.03 \\
\hline $\begin{array}{l}\text { Packaged heat pump, single- } \\
\text { speed reciprocating compressor, } \\
\text { PSC air-handler motor }\end{array}$ & 10 & 9.1 & 6.8 & 0.01 & 0.03 \\
\hline $\begin{array}{l}\text { Room air conditioner, louvered } \\
\text { sides, cooling only, single-speed } \\
\text { compressor, PSC fan motor, } \\
<20,000 \text { Btu/hr (after 1991) }\end{array}$ & & 9.75 & & 0.01 & 0.03 \\
\hline $\begin{array}{l}\text { Room air conditioner, louvered } \\
\text { sides, cooling only, single-speed } \\
\text { compressor, PSC fan motor, } \\
\geq 20,000 \text { Btu/hr (after 1991) }\end{array}$ & & 8.5 & & 0.01 & 0.03 \\
\hline
\end{tabular}


Table 8 continued. Default Air Conditioning and Heat-pump Efficiencies

\begin{tabular}{|c|c|c|c|c|c|}
\hline \multirow{2}{*}{$\begin{array}{l}\text { Type of Air-Conditioning or } \\
\text { Heat-Pump Equipment }\end{array}$} & \multirow{2}{*}{$\begin{array}{l}\text { Base } \\
\text { SEER }\end{array}$} & \multirow{2}{*}{$\begin{array}{l}\text { Base } \\
\text { EER }\end{array}$} & \multirow{2}{*}{$\begin{array}{l}\text { Base } \\
\text { HSPF }\end{array}$} & \multicolumn{2}{|c|}{ Maintenance Factor (M) } \\
\hline & & & & $\begin{array}{c}\text { Annual } \\
\text { Professional } \\
\text { Maintenance }\end{array}$ & $\begin{array}{c}\text { Seldom or } \\
\text { Never } \\
\text { Maintained }\end{array}$ \\
\hline $\begin{array}{l}\text { Room air conditioner, louvered } \\
\text { sides, cooling only, single-speed } \\
\text { compressor, PSC fan motor, } \\
(1981-1991)\end{array}$ & & 7.5 & & 0.01 & 0.03 \\
\hline $\begin{array}{l}\text { Room air conditioner, louvered } \\
\text { sides, cooling only, single-speed } \\
\text { compressor, PSC fan motor, } \\
\text { (before 1981) }\end{array}$ & & 6.5 & & 0.01 & 0.03 \\
\hline $\begin{array}{l}\text { Room electric heat pump, } \\
\text { louvered sides, single-speed } \\
\text { compressor, PSC fan motor, } \\
<20,000 \mathrm{Btu} / \mathrm{hr}\end{array}$ & & 9 & & 0.01 & 0.03 \\
\hline $\begin{array}{l}\text { Room electric heat pump, } \\
\text { louvered sides, single-speed } \\
\text { compressor, PSC fan motor, } \\
\geq 20,000 \mathrm{Btu} / \mathrm{hr}\end{array}$ & & 8.5 & & 0.01 & 0.03 \\
\hline Direct evaporative cooling & & 25 & & 0.02 & 0.05 \\
\hline
\end{tabular}


For houses with air ducts, the Pre-Retrofit Case shall be modeled using data collected through visual inspections, physical measurements, and duct-leakage testing. Default values for duct leakage shall not be used. Duct-blaster testing shall be conducted in accordance with ASTM E1554 (ASTM 1994). Tracer gas testing of the air distribution system is encouraged when possible, and shall be conducted in accordance with NREL Performance Test Practices for duct systems (Hancock et al. 2002).

If the simulation tool does not permit the input of detailed duct specifications, then two values (one for heating, one for cooling) of seasonal distribution system efficiency (DSE) shall be estimated and applied to the heating and cooling system efficiencies to represent expected energy losses from ducts. The DSE values shall be estimated using the procedures in the Draft ASHRAE Standard 152P (ASHRAE 2001).

For houses with hydronic space-heating or space-cooling systems, a distribution efficiency of 95\% shall be applied to the appliance efficiency, representing a small amount of energy loss through the pipes.

\section{Domestic Hot Water}

To the extent possible, the specifications of the domestic hot water system in the Pre-Retrofit Case shall be based on audits, design specifications, physical measurements, and test data. Published data from the manufacturer provides the most reliable estimate of energy factor, because in-situ testing introduces several uncontrolled variables (such as water use and ambient temperature) that usually make a reliable measurement of standby loss impossible. The procedures to measure recovery efficiency and standby losses described by LBNL (Szydlowski and Cleary 1988) may be used in conjunction with the NREL tank loss spreadsheet (www.eere.energy.gov/buildings/building america/docs/tankloss.xls) to give a rough estimate of the energy factor (EF). If EF of the equipment cannot be determined through measurement or examination of the published performance data, the default specifications in Table 9 may be used, with age and maintenance adjustments in accordance with Equation 3. These defaults were largely derived from technical support documents for the federal appliance standard for water heaters (DOE 2000a).

\section{Equation 3: $\quad \mathrm{EF}=($ Base EF $) *(1-\mathrm{M})^{\text {age }}$}

Where:

Base EF = Typical efficiency of Pre-Retrofit equipment when purchased

$\mathrm{M}=$ Maintenance Factor

Age $=$ Age of equipment in years . 
Table 9. Default DHW Energy Factors, Known Equipment Characteristics; "Gas" Refers to Either Natural Gas or Propane

\begin{tabular}{|c|c|c|c|}
\hline \multirow{2}{*}{ Type of Water-Heating Equipment } & \multirow{2}{*}{$\begin{array}{l}\text { Base } \\
\text { Energy } \\
\text { Factor } \\
\text { (EF) }\end{array}$} & \multicolumn{2}{|c|}{ Maintenance Factor (M) } \\
\hline & & $\begin{array}{c}\text { Annual } \\
\text { Professional } \\
\text { Maintenance }\end{array}$ & $\begin{array}{l}\text { Seldom or } \\
\text { Never } \\
\text { Maintained }\end{array}$ \\
\hline $\begin{array}{l}\text { Gas water heater, } 40 \text {-gallon tank, pilot } \\
\text { light, natural-draft combustion, poorly } \\
\text { insulated, no heat traps, poor heat } \\
\text { recovery from flue }\end{array}$ & 0.45 & 0.005 & 0.01 \\
\hline $\begin{array}{l}\text { Gas water heater, } 40 \text {-gallon tank, pilot } \\
\text { light, natural-draft combustion, } 1 \text {-in. } \\
\text { insulation, no heat traps, standard flue } \\
\text { baffling }\end{array}$ & 0.54 & 0.005 & 0.01 \\
\hline $\begin{array}{l}\text { Gas water heater, } 40 \text {-gallon tank, } \\
\text { intermittent ignition, forced draft } \\
\text { combustion, } 3 \text {-in. insulation, heat traps, } \\
\text { enhanced flue baffling, flue/vent } \\
\text { dampers }\end{array}$ & 0.64 & 0.005 & 0.01 \\
\hline Gas instantaneous water heater & 0.80 & 0.005 & 0.01 \\
\hline $\begin{array}{l}\text { Oil water heater, 32-gallon tank, } \\
\text { intermittent ignition, forced-draft } \\
\text { combustion, poorly insulated, no heat } \\
\text { traps, poor heat recovery from flue }\end{array}$ & 0.53 & 0.005 & 0.01 \\
\hline $\begin{array}{l}\text { Oil water heater, 32-gallon tank, } \\
\text { interrupted ignition, forced-draft } \\
\text { combustion, } 3 \text {-in. insulation, heat traps, } \\
\text { enhanced flue baffling }\end{array}$ & 0.61 & 0.005 & 0.01 \\
\hline $\begin{array}{l}\text { Electric water heater, } 50 \text {-gallon tank, } \\
\text { poorly insulated, no heat traps }\end{array}$ & 0.79 & 0.001 & 0.002 \\
\hline $\begin{array}{l}\text { Electric water heater, } 50 \text {-gallon tank, } \\
1.5 \text {-in. insulation, heat traps. }\end{array}$ & 0.87 & 0.001 & 0.002 \\
\hline $\begin{array}{l}\text { Electric water heater, } 50 \text {-gallon tank, } 3- \\
\text { in. insulation, heat traps. }\end{array}$ & 0.90 & 0.001 & 0.002 \\
\hline Electric instantaneous water heater & 1.00 & 0 & 0 \\
\hline
\end{tabular}


The NREL Performance Analysis Spreadsheet calculates the correct DHW inputs for the TRNSYS computer program, including standby heat loss coefficient (UA), given basic equipment characteristics (EF, RE, etc) (Burch and Erickson 2004). It can be found on the Building America Web site in the section for building scientists (www.eere.energy.gov/buildings/building america/pa resources.html).

Four major end uses have been identified for domestic hot water: showers, sinks, dishwasher, and clothes washer (Table 10). For showers and sinks, the specified volume is the same as the value defined for the Benchmark and represents the combined volume of hot and cold water. For clothes washers and dishwashers, the Appliance and DHW Spreadsheet developed by NREL shall be used to estimate the Pre- and Post-Retrofit hot water consumption based on standard operating conditions and information listed on the EnergyGuide label. If no EnergyGuide label is available, then the default values of energy factor for dishwashers (Table 11) or modified energy factor (MEF) for clothes washers (Table 12) shall be used for the Pre-Retrofit Case.

Table 10. Default Domestic Hot Water Consumption by End-use

\begin{tabular}{|l|c|c|}
\hline \multicolumn{1}{|c|}{ End Use } & $\begin{array}{c}\text { End-Use Water } \\
\text { Temperature }\end{array}$ & Default Water Usage \\
\hline Dishwasher & N/A & Calculated using EF in Table 10 \\
\hline Clothes Washer & N/A & Calculated using MEF in Table 11 \\
\hline Shower and Bath & $105^{\circ} \mathrm{F}$ & $17.5+5.83 \times \mathrm{N}_{\mathrm{br}}$ gal $/$ day (Hot + Cold) \\
\hline Sinks & $105^{\circ} \mathrm{F}$ & $12.5+4.16 \times \mathrm{N}_{\mathrm{br}}$ gal/day (Hot + Cold) \\
\hline
\end{tabular}

Table 11. Default Dishwasher Characteristics

\begin{tabular}{|l|c|}
\hline \multicolumn{1}{|c|}{ Equipment Characteristics } & $\begin{array}{c}\text { Energy Factor } \\
\text { (load/kWh) }\end{array}$ \\
\hline $\begin{array}{l}\text { Power dry optional, multi-tier spray device, load-size and soil- } \\
\text { level controls }\end{array}$ & 0.6 \\
\hline $\begin{array}{l}\text { Power dry optional, multi-tier spray device, no load-size or soil- } \\
\text { level controls }\end{array}$ & 0.43 \\
\hline $\begin{array}{l}\text { Power dry always, single-tier spray device, no load-size or soil- } \\
\text { level controls }\end{array}$ & 0.24 \\
\hline
\end{tabular}


Table 12. Default Standard Size $\left(\sim 2.5 \mathrm{ft}^{3}\right)$ Clothes Washer Characteristics

\begin{tabular}{|l|c|}
\hline \multicolumn{1}{|c|}{ Equipment Characteristics } & $\begin{array}{c}\text { Modified Energy } \\
\left.\text { Factor ( } \mathrm{ft}^{3} / \mathbf{k W h}\right)\end{array}$ \\
\hline $\begin{array}{l}\text { Horizontal axis, cold rinse option, automatic fill, thermostatically } \\
\text { controlled mixing valve, improved water extraction }\end{array}$ & 1.62 \\
\hline $\begin{array}{l}\text { Vertical axis, cold rinse option, automatic fill, thermostatically } \\
\text { controlled mixing valve, improved water extraction }\end{array}$ & 1.02 \\
\hline $\begin{array}{l}\text { Vertical axis, cold rinse option, water level option, standard } \\
\text { mixing valve }\end{array}$ & 0.64 \\
\hline Vertical axis, no cold rinse option, no water level option & 0.47 \\
\hline
\end{tabular}

The hourly hot water use profiles for individual end-uses in existing homes (Figures 1 through 3) are the same as the profiles recommended by Building America for analyzing new construction (Hendron 2005). For software tools that do not accept this level of detail, or if no DHW end-use improvements have been made to the house, then the combined hourly hot water profile may be used (Figure 4).

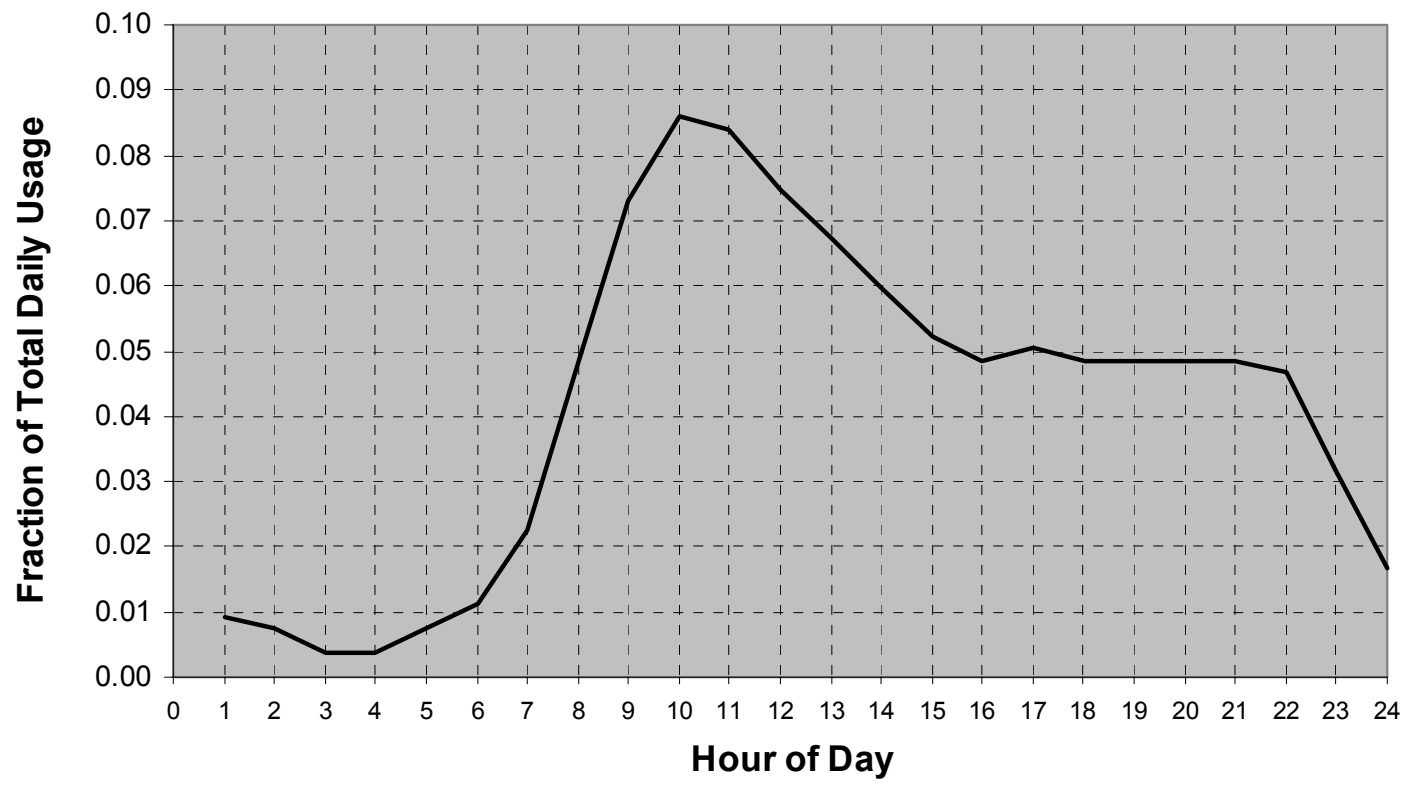

Figure 1. Clothes washer hot water use profile 


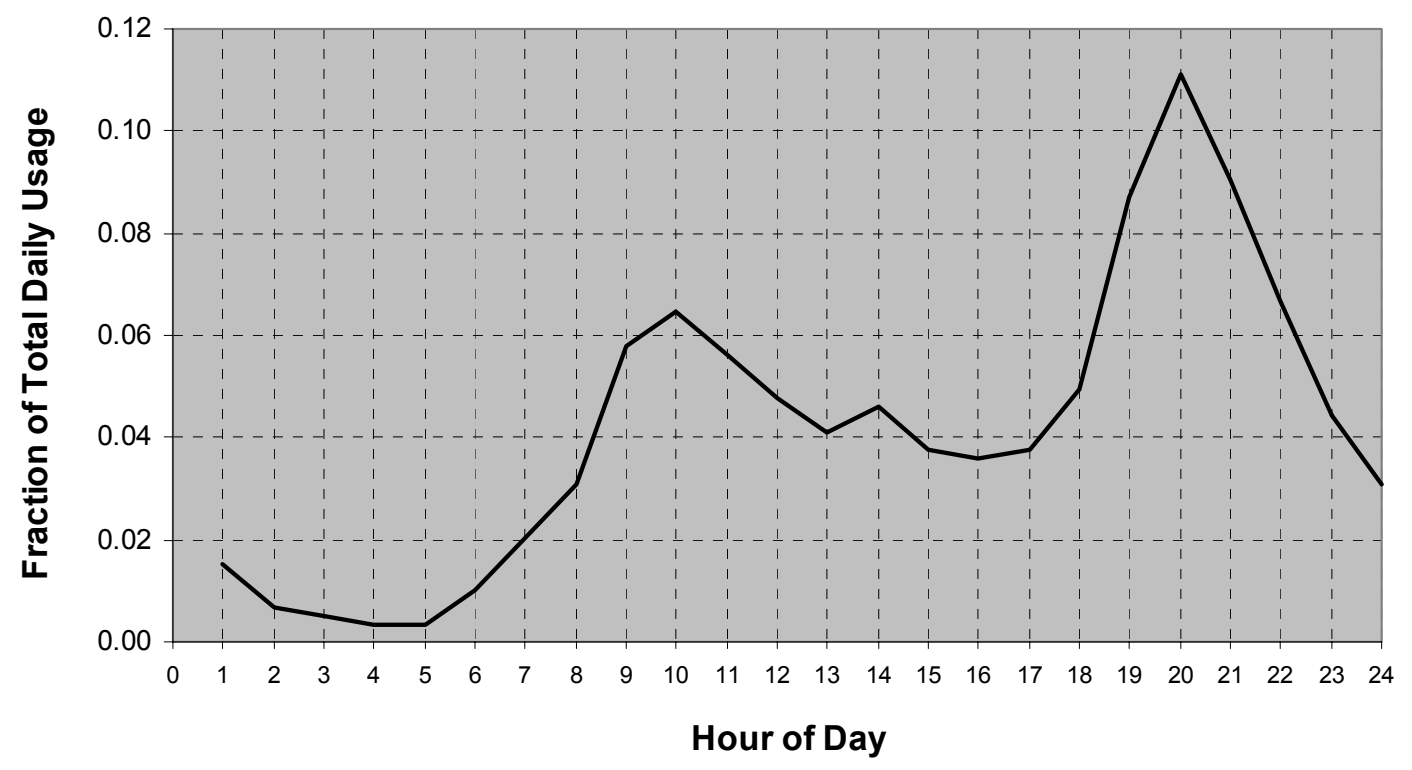

Figure 2. Dishwasher hot water use profile

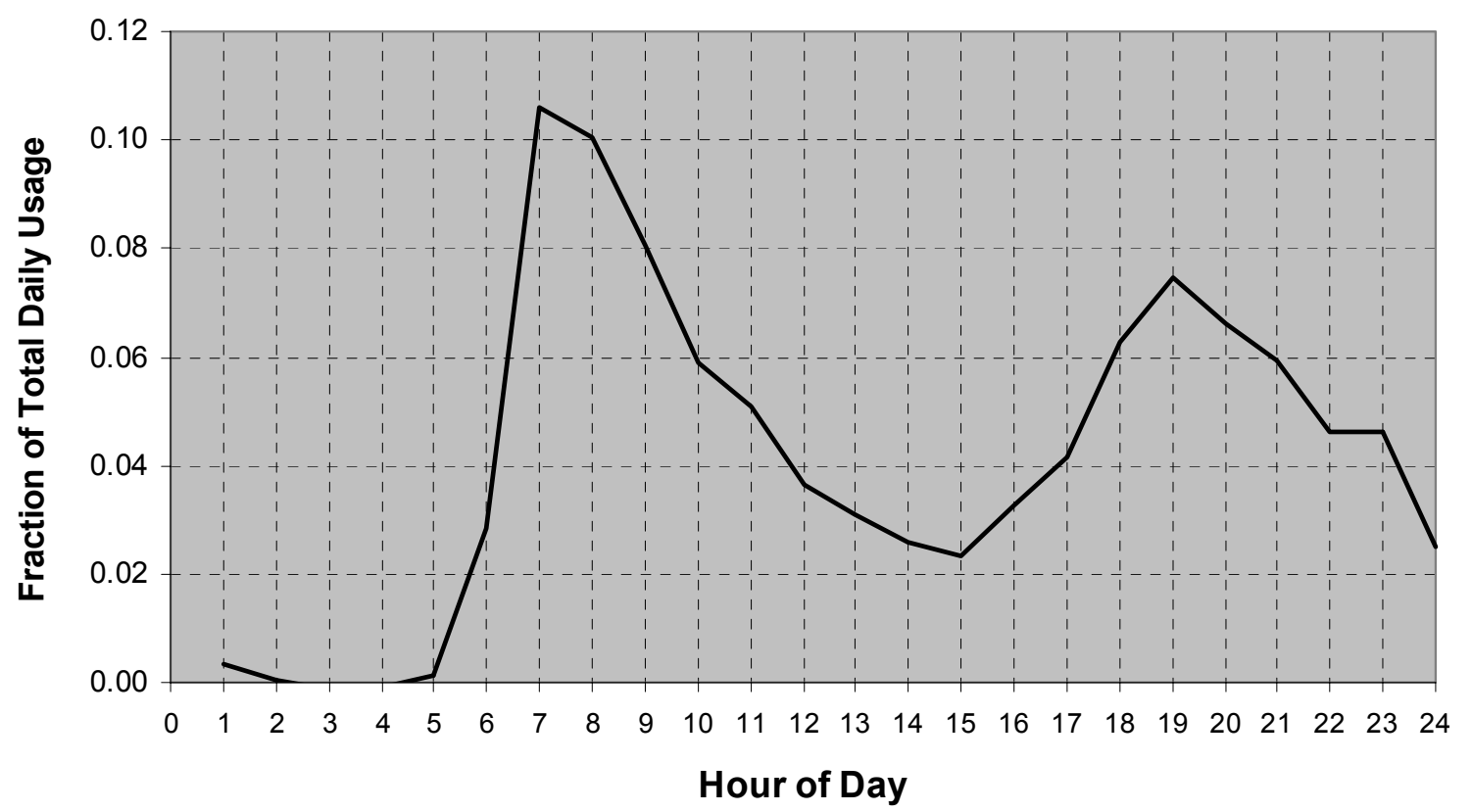

Figure 3. Shower, bath, and sink hot water use profile 


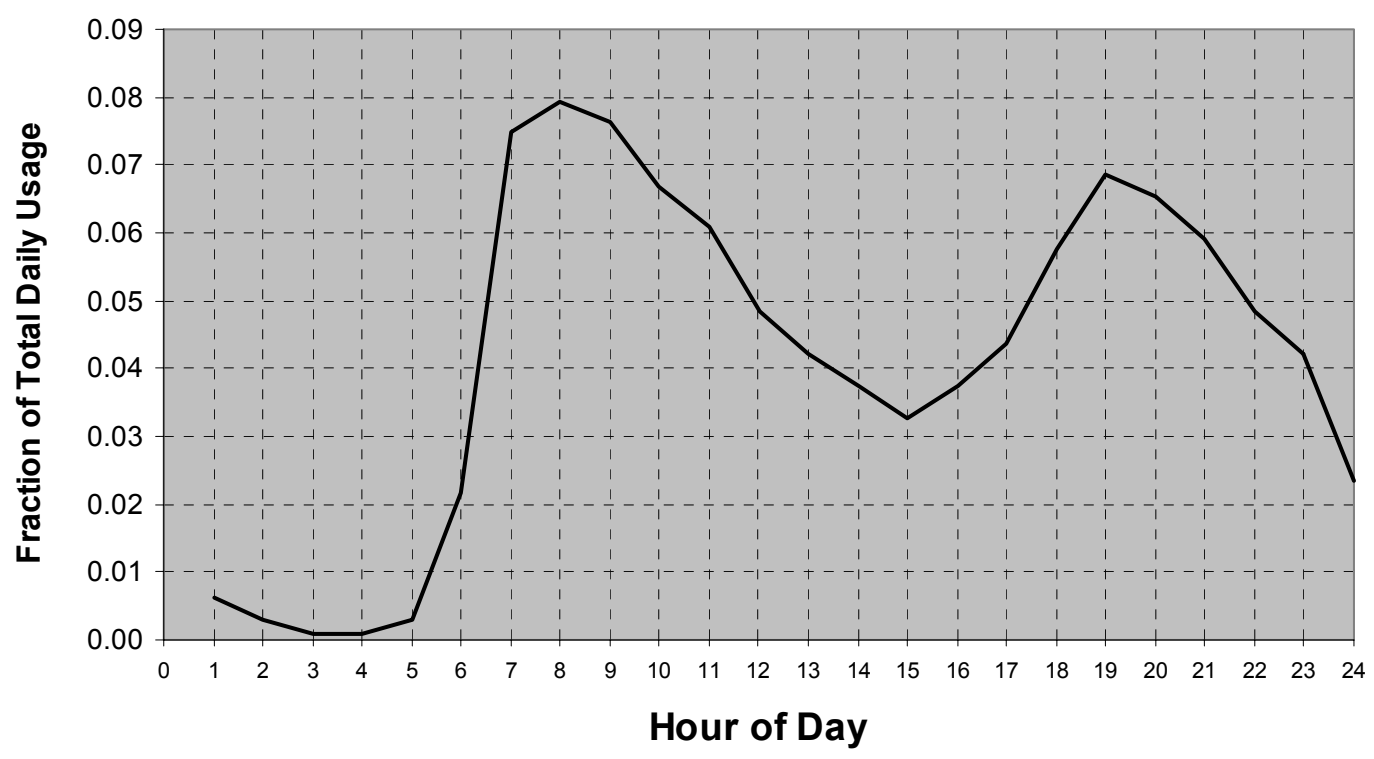

Figure 4. Combined domestic hot water use profile

The mains water temperature, which is an important driver of water-heating energy, varies significantly depending on the location and time of year. Equation 4, based on TMY2 data for the geographic location of the existing house, shall be used to estimate the daily mains water temperature for both the Pre- and Post-Retrofit Cases. Because temperature changes between the mains and the DHW inlet and/or cold water fixtures are not factored into this equation, it is currently assumed that the mains temperature and the cold water temperature inside the house are approximately the same. The derivation of the mains temperature equation is discussed in more detail in the Benchmark report (Hendron 2005).

Equation 4: $T_{\text {mains }}=\left(T_{\text {amb,avg }}+\right.$ offset $)+$ ratio $*\left(\Delta T_{\text {amb,max }} / 2\right) * \sin (0.986 *($ day\# 15 - lag) - 90)

where:

\begin{tabular}{|c|c|c|}
\hline $\mathrm{T}_{\text {mains }}$ & & $\begin{array}{l}\text { mains temperature }\left({ }^{\circ} \mathrm{F}\right) \text {, assumed equal to the cold water } \\
\text { supply temperature }\end{array}$ \\
\hline $\mathrm{T}_{\text {amb,avg }}$ & $=$ & annual average ambient air temperature $\left({ }^{\circ} \mathrm{F}\right)$ \\
\hline$\Delta \mathrm{T}_{\mathrm{amb}, \max }$ & $=$ & $\begin{array}{l}\text { maximum difference between monthly average ambient } \\
\text { temperatures (e.g., } T_{\text {amb,avg,july }}-T_{\text {amb,avg,january }}\left({ }^{\circ} \mathrm{F}\right)\end{array}$ \\
\hline 0.986 & $=$ & degrees/day $(360 / 365)$ \\
\hline day\# & $=$ & Julian day of the year (1-365) \\
\hline offset & $=$ & $6^{\circ} \mathrm{F}$ \\
\hline ratio & $=$ & $0.4+0.01\left(\mathrm{~T}_{\mathrm{amb}, \mathrm{avg}}-44\right)$ \\
\hline lag & $=$ & $35-1.0\left(\mathrm{~T}_{\mathrm{amb}, \mathrm{avg}}-44\right)$. \\
\hline
\end{tabular}




\section{Air Infiltration and Ventilation}

The effective leakage area for the Pre-Retrofit Case shall be calculated based on blower door testing conducted in accordance with ASTM E779-03 (ASTM 2003). If the whole-house simulation tool being used cannot calculate hourly infiltration based on effective leakage area, an annual average natural infiltration rate may be used based on the guidelines in ASHRAE Standard 119 (ASHRAE 1988), Section 5, and ASHRAE Standard 136 (ASHRAE 1993), Section 4. It is recommended that blower door measurements be supplemented with tracer gas testing when possible.

Additional air exchange resulting from mechanical ventilation shall be assumed for the model of the Pre-Retrofit Case, if it does not meet the ventilation guidelines of ASHRAE Standard 62.22004 (ASHRAE 2004b) for existing homes based on natural infiltration plus any existing mechanical ventilation system. This "supplemental" mechanical ventilation shall be calculated using Equation 5, which is based on a simple continuous exhaust fan designed to raise the total ventilation rate to the minimum values specified in Equation 4.1a of ASHRAE Standard 62.22004, taking into account any infiltration credit allowed under Section 4.1.3. Supplemental mechanical ventilation shall be combined with the actual ventilation and natural infiltration in accordance with Section 4.4 of ASHRAE Standard 136 to determine an approximate combined effective air-change rate. The fan energy use associated with supplemental mechanical ventilation for the Pre-Retrofit shall be calculated using Equation 6 and added to the energy used by any actual ventilation fan present in the house.

Equation 5: Qsup $=[0.01 \times$ FFA + 7.5 $\times(\mathrm{Nbr}+1)]-[$ AI $\times$ CFA $\times$ H $/ 60-2 \times$ FFA $/ 100] / 2$ where:

$$
\begin{aligned}
Q_{\text {sup }}= & \begin{array}{l}
\text { supplemental mechanical ventilation assumed for the Pre-Retrofit Case } \\
(\mathrm{cfm}) \text { not including interactions with natural infiltration }
\end{array} \\
\mathrm{FFA}= & \text { finished floor area }\left(\mathrm{ft}^{2}\right) \\
\mathrm{CFA}= & \begin{array}{l}
\text { conditioned floor area, including directly or indirectly conditioned } \\
\text { basements }\left(\mathrm{ft}^{2}\right)
\end{array} \\
\mathrm{H}= & \text { average height of one story }(\mathrm{ft}) \\
\mathrm{A}_{\mathrm{I}}= & \text { annual average air changes per hour resulting from natural infiltration } \\
& (\mathrm{ACH})
\end{aligned}
$$

\section{Equation 6: Supplemental ventilation fan energy $(\mathrm{kWh} / \mathrm{yr})=3.942 \times Q_{\text {sup. }}$}

\section{Lighting Equipment and Usage}

The total annual lighting budget for the Pre-Retrofit case shall be determined by conducting a detailed audit of light fixtures and bulbs inside and outside the house. Operating hours may be determined through long-term monitoring or by conducting occupant interviews or surveys. If 
reliable estimates of operating hours cannot be obtained or calculated, then the default operating hours estimated in the Navigant study may be used (Table 13).

Table 13. Default Lighting Operating Hours for Common Room Types (Navigant 2002)

\begin{tabular}{|l|c|l|c|}
\hline Room Type & $\begin{array}{c}\text { Operation } \\
\text { (Hours/day/room) }\end{array}$ & Room Type & $\begin{array}{c}\text { Operation } \\
\text { (Hours/day/room) }\end{array}$ \\
\hline Bathroom & 1.8 & Kitchen & 3.0 \\
\hline Bedroom & 1.1 & Living Room & 2.5 \\
\hline Closet & 1.1 & Office & 1.7 \\
\hline Dining Room & 2.5 & Outdoor & 2.1 \\
\hline Family Room & 1.8 & Utility Room & 2.0 \\
\hline Garage & 1.5 & Other & 0.8 \\
\hline Hall & 1.5 & & \\
\hline
\end{tabular}

The annual average normalized daily load shape for interior lighting use is shown in Figure 5 and is the same profile used by Building America in the context of new construction. This load shape is also used for exterior and garage lighting. Monthly variations in load shape and lighting energy use resulting from changes in the length of days can be accounted for, as long as the variation is applied to all the simulation models and total annual energy use remains the same. Other factors may also be considered, including day types (weekday vs. weekend), occupancy types (day-use vs. non-day-use or "nuclear" vs. "yuppie"), season (summer vs. winter), and room types (living area vs. bedroom area). Individual normalized profiles can be "rolled up" to various levels of detail appropriate to the simulation model. An example of one detailed set of profiles developed by NREL is shown in Figure 6. Other profiles are included in spreadsheets available on the Building America Web site (www.eere.energy.gov/buildings/building_america/pa resources.html). 


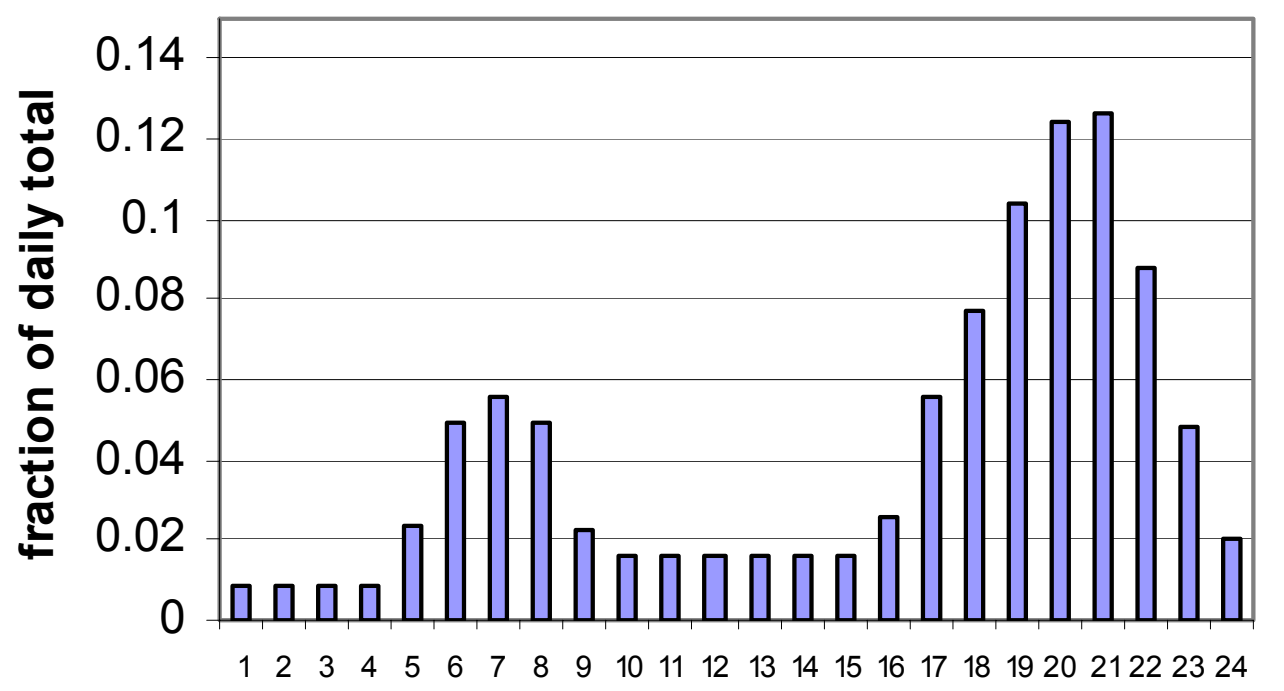

Figure 5. Annual average interior lighting profile (built up from detailed profiles)

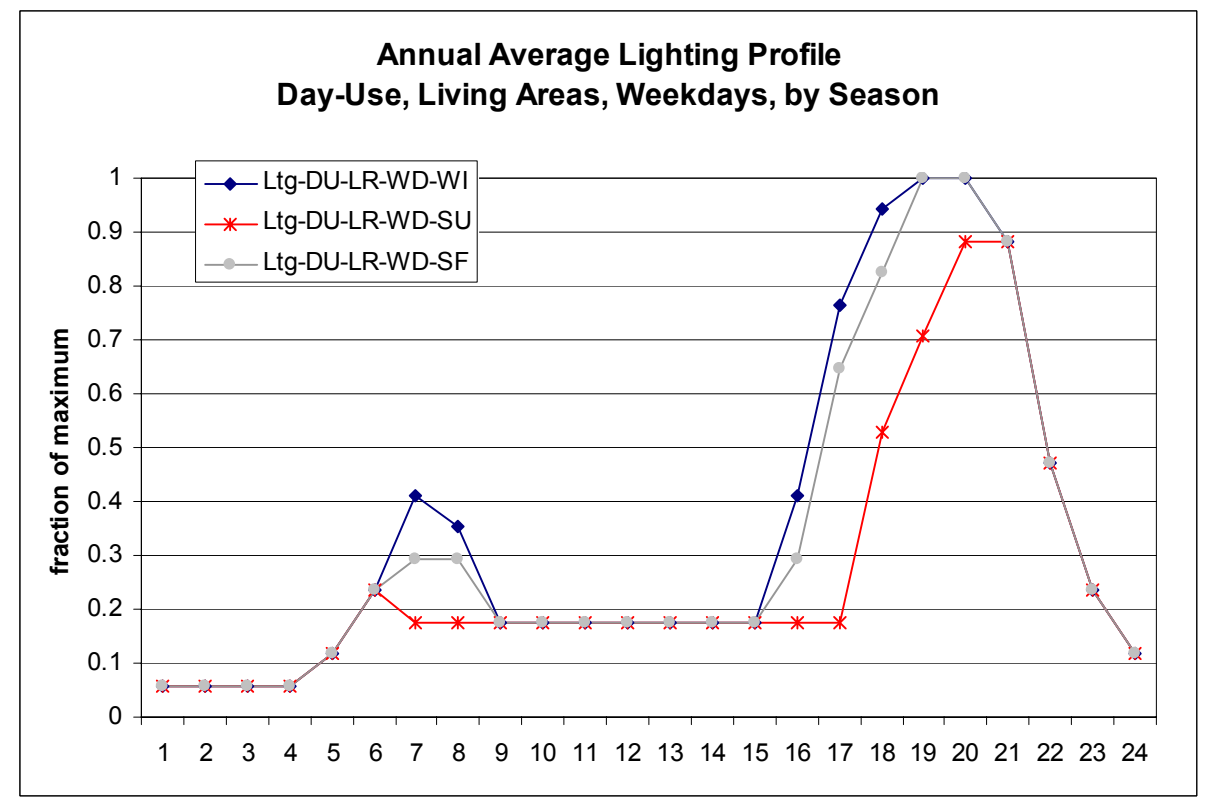

Figure 6. Example of a detailed lighting profile (expressed as fraction of peak daily lighting energy)

\section{Appliances and Miscellaneous Electric Loads}

As with lighting, several characteristics must be defined for appliances and miscellaneous electric loads (MELs): the amount of the load, the schedule of the load, the location of the load, the fraction of the load that becomes a sensible heat gain, and the fraction of the load that becomes a latent heat gain. 
To the extent possible, actual specifications for all major appliances should be obtained through inspection. Spot electricity measurements may be performed for loads that are relatively constant when operating, such as refrigerators and freezers. A more standardized procedure for calculating average daily electricity use for refrigerators was developed by LBNL (Szydlowski and Cleary 1988). If EnergyGuide labels are not used, it is important that the same refrigerator audit procedures are used for both the Pre- and Post-Retrofit cases to ensure a fair comparison.

If EnergyGuide labels are available for dishwashers or clothes washers, then the BA Analysis Spreadsheet shall be used to estimate annual energy use. If EnergyGuide labels cannot be located or do not exist for certain major appliances (e.g., ovens and clothes dryers), the default energy factors in Tables 11 through 12 and 14 through 17 shall be used. These defaults were derived from historical appliance efficiency studies (Wenzel et al. 1997, DOE 2004b, EPRI 1986) and technical support documents for recent changes to Federal appliance standards (DOE 1993, DOE 2000b). If the specific equipment type is not listed in the default tables, the efficiency may either be interpolated based on listed equipment or estimated using the original reference sources. The default efficiencies must be used in conjunction with the BA Analysis Spreadsheet to estimate annual electricity and hot water use.

Operating hours estimated through occupant surveys or interviews may be useful for determining the cost-effectiveness of replacing certain appliances and electronic equipment for a particular homeowner. However, the standard operating conditions specified for the Benchmark shall be used for the purpose of calculating and reporting whole-house energy savings for the PostRetrofit Case.

Table 14. Default Gas Clothes Dryer Characteristics (Assumes Typical 1990 Clothes Washer Capacity and Remaining Moisture Content)

\begin{tabular}{|l|c|}
\hline \multicolumn{1}{|c|}{ Equipment Characteristics } & $\begin{array}{c}\text { Energy } \\
\text { Factor } \\
\text { (Ib/kWh) }\end{array}$ \\
\hline $\begin{array}{l}\text { Cool-down mode, intermittent ignition, automatic termination } \\
\text { control, improved door seal, well insulated }\end{array}$ & 2.67 \\
\hline $\begin{array}{l}\text { Cool-down mode, intermittent ignition, timer control, improved door } \\
\text { seal, well insulated }\end{array}$ & 2.40 \\
\hline $\begin{array}{l}\text { No cool-down mode, pilot light, timer control, poor door seal, } \\
\text { poorly insulated }\end{array}$ & 2.00 \\
\hline
\end{tabular}


Table 15. Default Electric Clothes Dryer Characteristics (Assumes Typical 1990 Clothes Washer Capacity and Remaining Moisture Content)

\begin{tabular}{|l|c|}
\hline \multicolumn{1}{|c|}{ Equipment Characteristics } & $\begin{array}{c}\text { Energy Factor } \\
\text { (lb/kWh) }\end{array}$ \\
\hline $\begin{array}{l}\text { Cool-down mode, automatic termination control, improved door } \\
\text { seal, well insulated }\end{array}$ & 2.75 \\
\hline $\begin{array}{l}\text { No cool-down mode, timer control, poor door seal, poorly } \\
\text { insulated }\end{array}$ & 2.60 \\
\hline
\end{tabular}

Table 16. Default Gas Oven / Cooktop Characteristics

\begin{tabular}{|l|l|l|}
\hline \multicolumn{1}{|c|}{ Equipment Characteristics } & Energy Factor & $\begin{array}{l}\text { Annual Gas Energy } \\
\text { (therms/yr) }\end{array}$ \\
\hline $\begin{array}{l}\text { Cooktop: intermittent ignition, sealed burner } \\
\text { Oven: spark ignition, not self cleaning, } \\
\text { improved door seals, reduced vent rate, high- } \\
\text { density insulation }\end{array}$ & $\begin{array}{l}\text { Cooktop: } 42.0 \% \\
\text { Oven: } 6.2 \%\end{array}$ & $\begin{array}{l}\text { Cooktop: } 17 \\
\text { Oven: } 18\end{array}$ \\
\hline $\begin{array}{l}\text { Cooktop: intermittent ignition, open burner } \\
\text { Oven: electric glo-bar ignition, self cleaning }\end{array}$ & $\begin{array}{l}\text { Cooktop: } 40.0 \% \\
\text { Oven: } 5.8 \%\end{array}$ & $\begin{array}{l}\text { Cooktop: } 18 \\
\text { Oven: } 19(+80 \mathrm{kWh})\end{array}$ \\
\hline $\begin{array}{l}\text { Cooktop: pilot lights } \\
\text { Oven: pilot light, not self-cleaning, standard } \\
\text { door seals, standard vent rate, standard } \\
\text { insulation }\end{array}$ & $\begin{array}{l}\text { Cooktop: } 18.8 \% \\
\text { Oven: } 3.5 \%\end{array}$ & $\begin{array}{l}\text { Cooktop: } 39 \\
\text { Oven: } 36\end{array}$ \\
\hline
\end{tabular}


Table 17. Default Electric Oven / Cooktop Characteristics

\begin{tabular}{|l|l|l|}
\hline \multicolumn{1}{|c|}{ Equipment Characteristics } & Energy Factor & $\begin{array}{c}\text { Annual Electric } \\
\text { Energy (kWh/yr) }\end{array}$ \\
\hline $\begin{array}{l}\text { Cooktop: reflective pans, flat coil elements } \\
\text { Oven: self-cleaning, improved door seals }\end{array}$ & $\begin{array}{l}\text { Cooktop: } 77.7 \% \\
\text { Oven: } 10.2 \%\end{array}$ & $\begin{array}{l}\text { Cooktop: } 270 \\
\text { Oven: } 349\end{array}$ \\
\hline $\begin{array}{l}\text { Cooktop: solid disc elements } \\
\text { Oven: not self-cleaning, improved door seals, } \\
\text { reduced vent rate, high-density insulation }\end{array}$ & $\begin{array}{l}\text { Cooktop: } 74.2 \% \\
\text { Oven: } 12.1 \%\end{array}$ & $\begin{array}{l}\text { Cooktop: } 282 \\
\text { Oven: } 293\end{array}$ \\
\hline $\begin{array}{l}\text { Cooktop: non-reflective pans, rounded coil } \\
\text { elements } \\
\text { Oven: not self-cleaning, standard door seals, } \\
\text { standard vent rate, standard insulation }\end{array}$ & Cooktop: $73.7 \%$ & $\begin{array}{l}\text { Cooktop: } 284 \\
\text { Oven: } 326\end{array}$ \\
\hline
\end{tabular}

In most cases, Miscellaneous Electric Loads (MELs) shall be treated as a constant function of finished floor area, regardless of the actual MELs present in the Pre-Retrofit Case (Equation 7). A multiplier is applied if the house is located in one of the four most populated states as determined in the EIA Residential Energy Consumption Survey (RECS) (DOE 2001). Multipliers for these four states were estimated based on the final electric end-use regression equations developed for the 2001 RECS, substituting national average values for known housing characteristics and physical traits of the occupants (such as number of bedrooms, number of ceiling fans, and age of homeowner) and removing end-uses that are treated separately in this report (such as lighting and clothes dryer). The multiplier is 1.0 for most states because insufficient information is available about the magnitude of MELs in those states.

Equation 7: $\quad$ MEL $=\left(2803+0.316 \times\right.$ FFA $\left.+194 \times N_{b r}\right) \times F_{s}$,

where:

$$
\begin{aligned}
\text { MEL }= & \text { miscellaneous electric loads for the Pre-Retrofit Case }(\mathrm{kWh} / \mathrm{yr}) \\
\mathrm{FFA}= & \text { finished floor area }\left(\mathrm{ft}^{2}\right) \\
\mathrm{N}_{\mathrm{br}}= & \text { number of bedrooms } \\
\mathrm{F}_{\mathrm{s}}= & \text { state multiplier }(\text { New York }=0.82, \text { California }=0.77, \text { Florida }=0.94, \\
& \text { Texas }=1.11, \text { all others }=1.00) .
\end{aligned}
$$

Alternatively, if MEL improvements are included in the retrofit package, analysts may use the more detailed methodology developed for new construction (Hendron and Eastment 2006), which allows energy savings credit for replacement of small appliances and reduction of standby losses. This methodology is automated in the BA MEL Analysis Spreadsheet (www.eere.energy.gov/buildings/building_america/pa_resources.html). 
The fraction of end-use energy converted into internal sensible and latent load is shown in Table 18. Not all of the energy consumed by appliances is converted into internal load; much of the waste heat is exhausted to the outside or released down the drain in the form of hot water.

\section{Table 18. Default Internal Loads from Appliances and Small Electric End-uses in the Pre-Retrofit Case}

\begin{tabular}{|l|c|c|}
\hline \multicolumn{1}{|c|}{ Appliance } & $\begin{array}{c}\text { Sensible Load } \\
\text { Fraction }\end{array}$ & Latent Load Fraction \\
\hline Refrigerator & 1.00 & 0.00 \\
\hline Freezer & 1.00 & 0.00 \\
\hline Clothes Washer & 0.80 & 0.00 \\
\hline Clothes Dryer (Electric) & 0.15 & 0.05 \\
\hline Clothes Dryer (Gas) & 1.00 (Electric) & 0.00 (Electric) \\
\hline Dishwasher (eight place settings) & 0.10 (Gas) & 0.05 (Gas) \\
\hline Range (Electric) & 0.60 & 0.15 \\
\hline Range (Gas) & 0.40 & 0.30 \\
\hline Plug-In Lighting & 0.30 & 0.20 \\
\hline Miscellaneous Electric Loads & 1.00 & 0.00 \\
\hline
\end{tabular}

The hourly, normalized load shape for combined residential equipment (Figure 7) is based on the results of the End-Use Load and Consumer Assessment Program (ELCAP) study of household electricity use in the Pacific Northwest (Pratt et al. 1989). In most situations this profile is adequate for simulating all electric and gas end-uses except space conditioning and hot water. However, because some individual end-use profiles are nearly constant (such as refrigerator and transformer loads) and some are highly dependent on time of day (such as the range and dishwasher), we have also developed a series of normalized hourly profiles for major appliances and MELs (Figures 8 through13. Numerical values associated with these profiles can be found in the BA Analysis Spreadsheet posted on the Building America web site (www.eere.energy.gov/buildings/building_america/pa_resources.html). The hourly profiles for machine energy usage in the clothes washer and dishwasher are identical to those provided earlier in the section on DHW (Figures 1 and 2). The profile for plug-in lighting is the same as the profile for hard-wired lighting presented in Figure 5. 
All hourly end-use profiles were taken from the ELCAP study, except the profile for Miscellaneous Electric Loads, which was derived by subtracting the energy consumption profiles for the major appliances from the combined profile for all equipment, assuming an all-electric, $1800-\mathrm{ft}^{2}$, three-bedroom house in Memphis, Tennessee. Because the MEL profile is based on a residual, it is susceptible to greater systematic errors and may be less realistic than the profiles for major appliances. These end-use profiles are the same as those used for analyzing new construction. Internal sensible and latent heat gains from appliances and plug loads shall be modeled using the same profiles used for end-use consumption. Appliance loads may be modeled in either the living spaces or bedroom spaces, depending on their location in the house.

Large end uses in the Pre-Retrofit Case that are not part of typical houses (such as swimming pools, Jacuzzis, and workshops) should not be explicitly included in the models for either the Pre- or Post-Retrofit Case. The efficiency of these end uses should be addressed in a separate analysis.

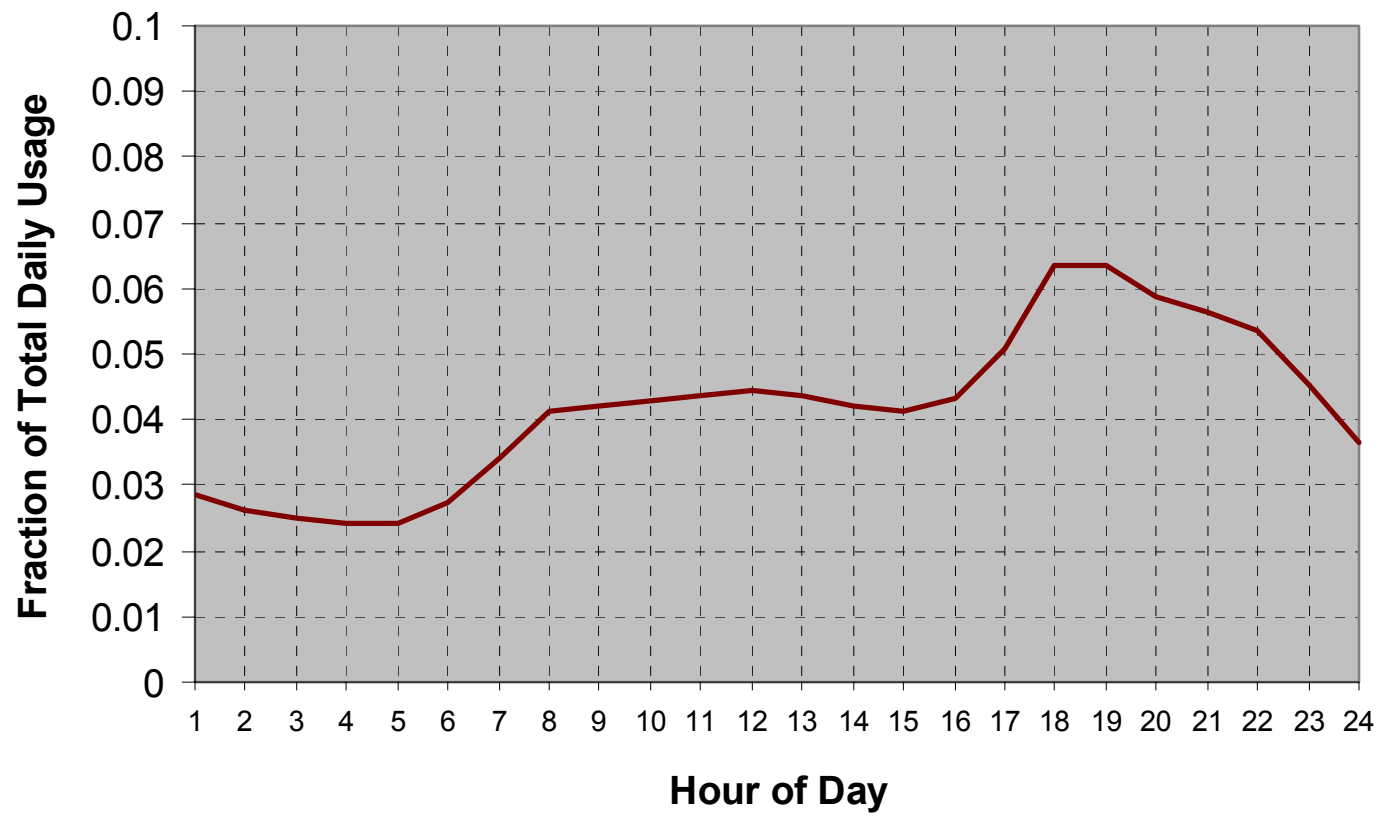

Figure 7. Total combined residential equipment profile (Pratt et al. 1989) 


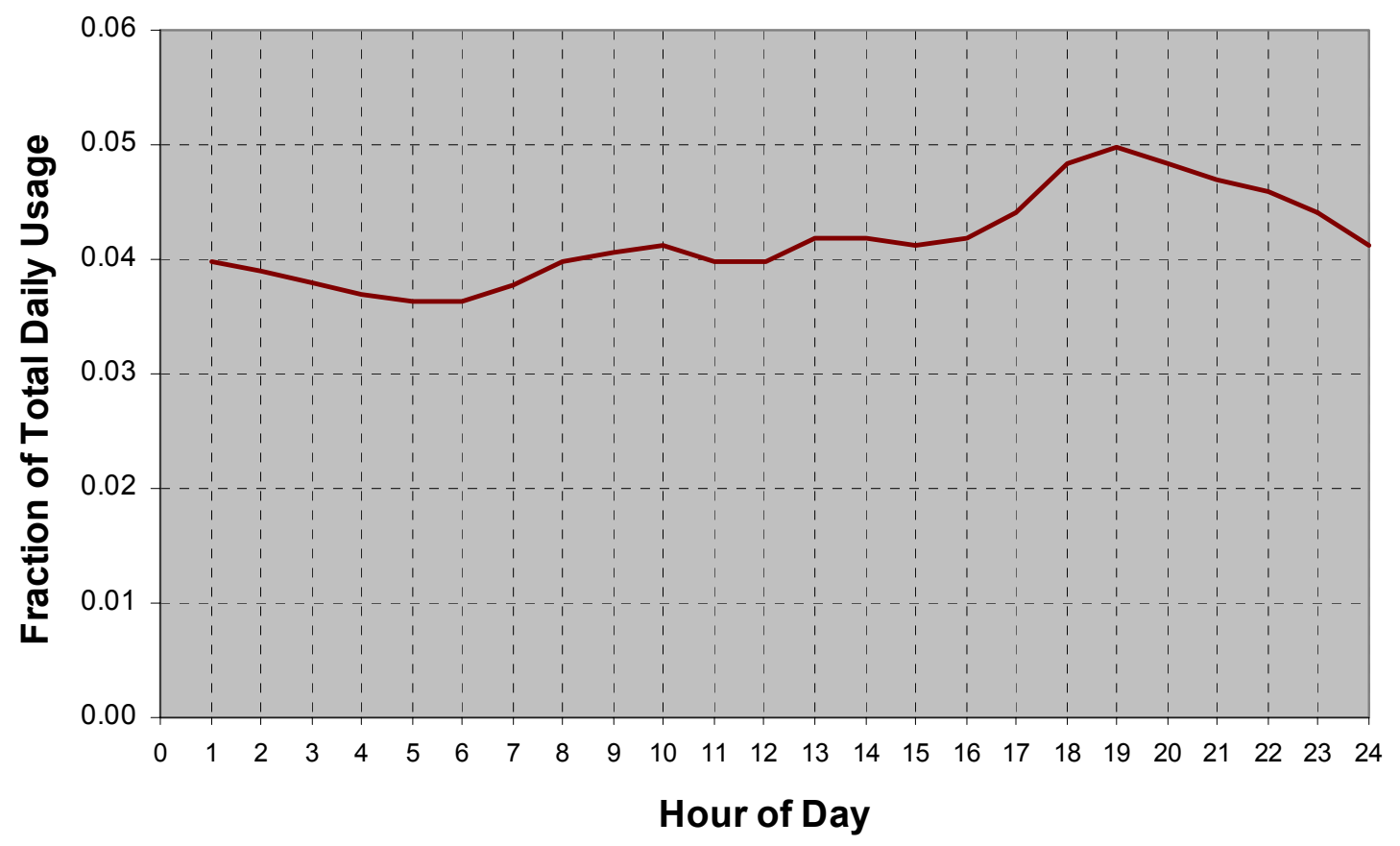

Figure 8. Refrigerator and freezer normalized energy-use profile (Pratt et al. 1989)

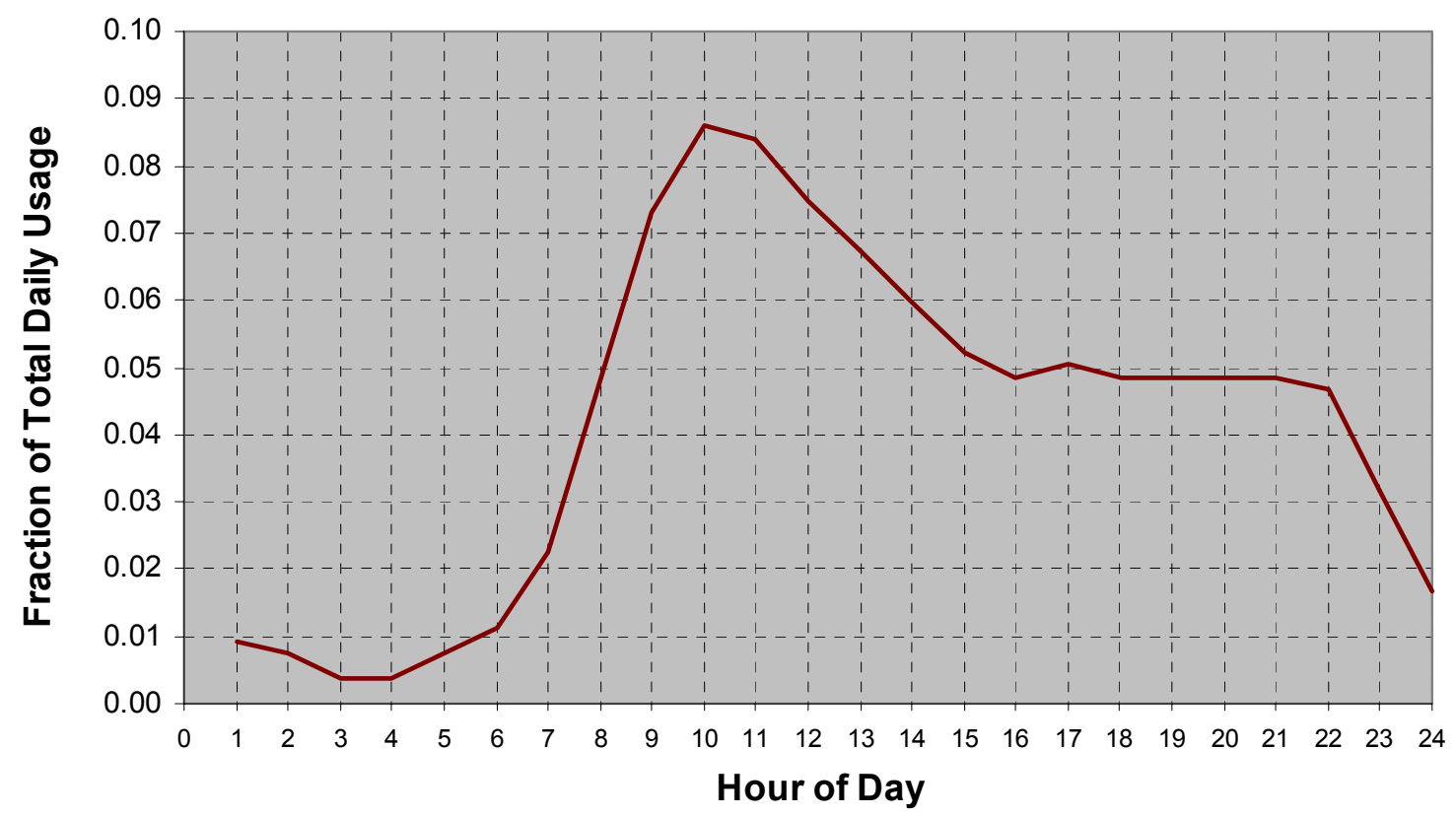

Figure 9. Clothes washer normalized machine energy-use profile (Pratt et al. 1989) 


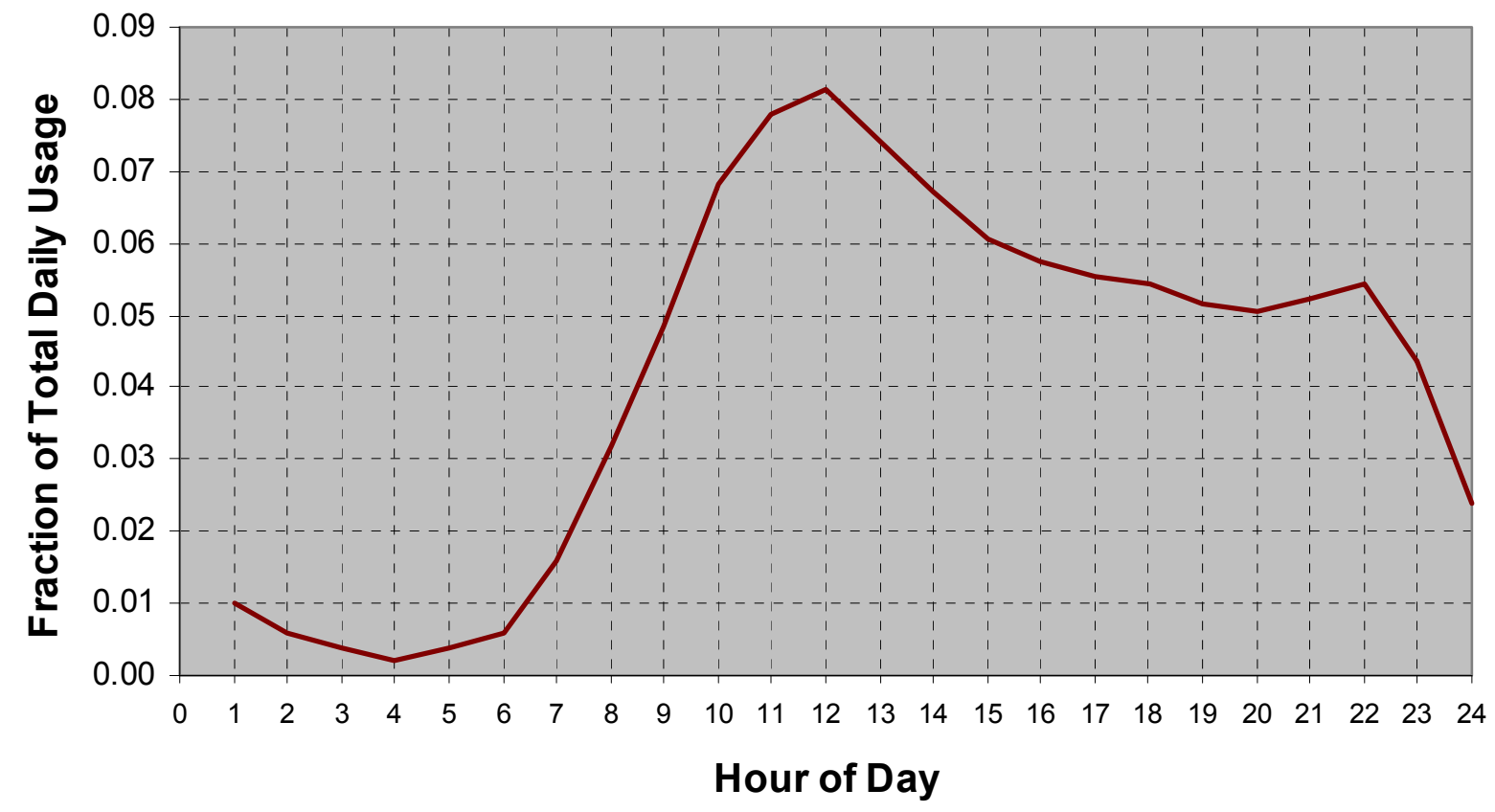

Figure 10. Clothes dryer normalized energy use profile (Pratt et al. 1989)

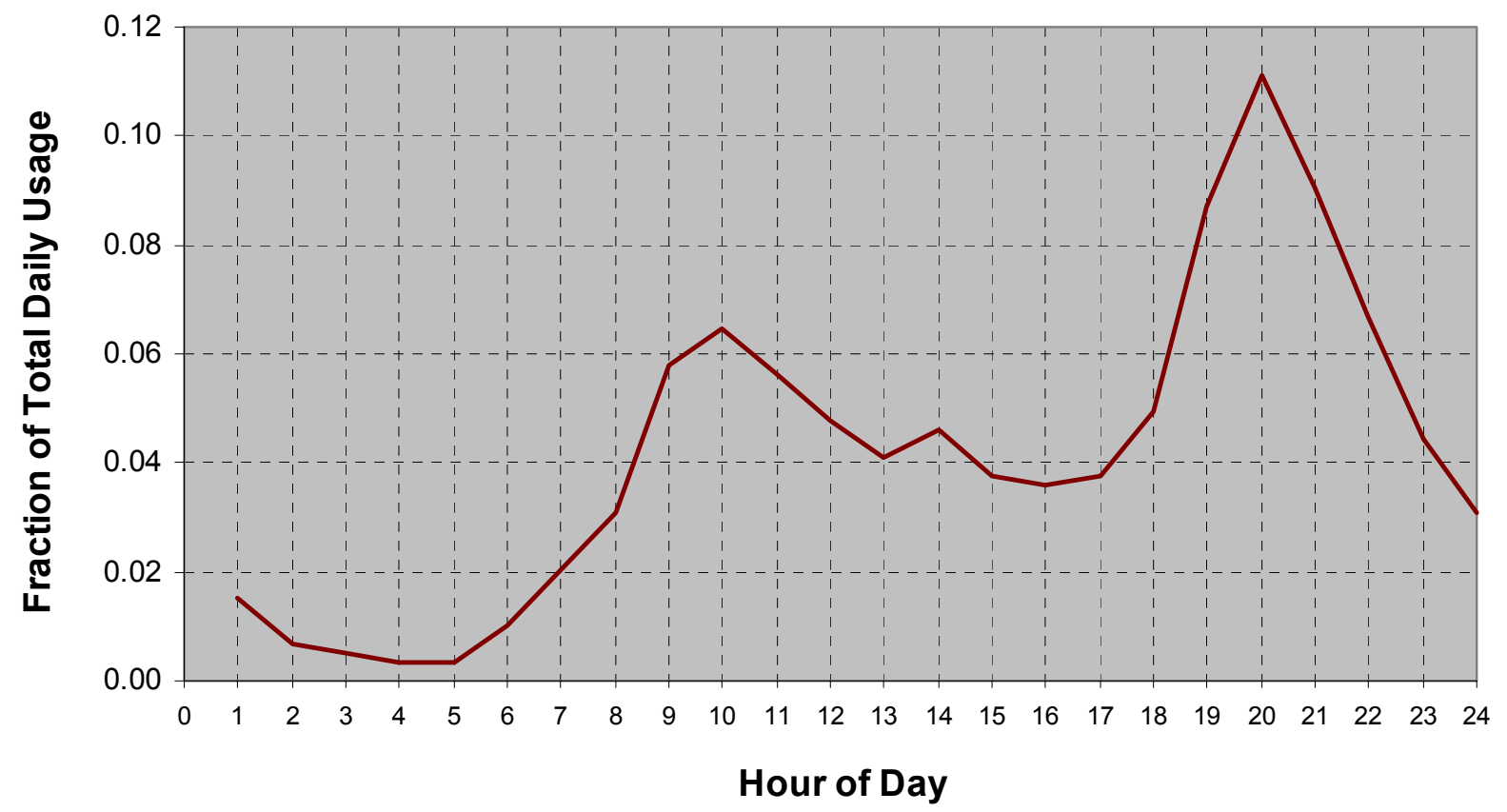

Figure 11. Dishwasher normalized energy use profile (Pratt et al. 1989) 


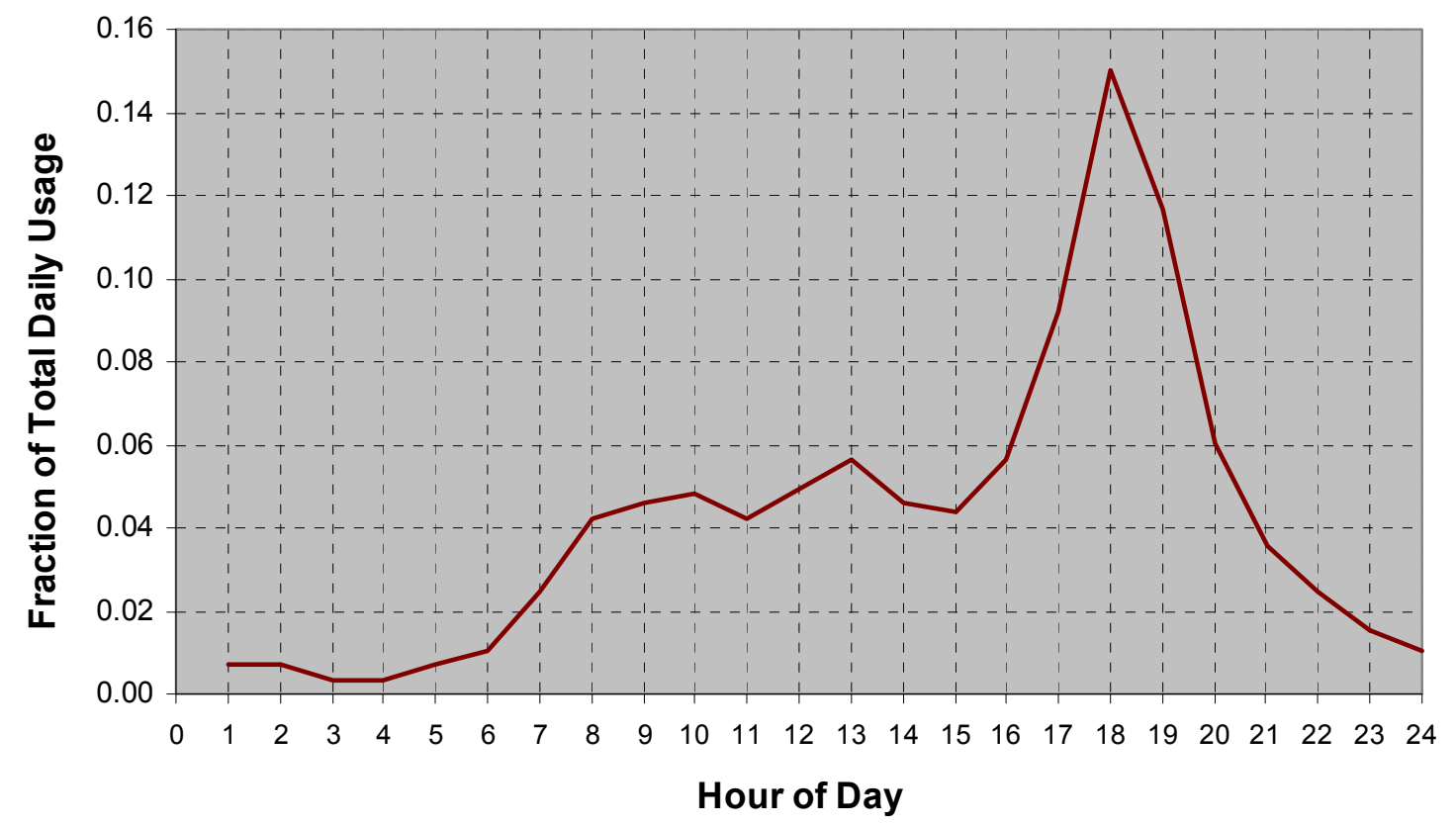

Figure 12. Range / oven normalized energy-use profile (Pratt et al. 1989)

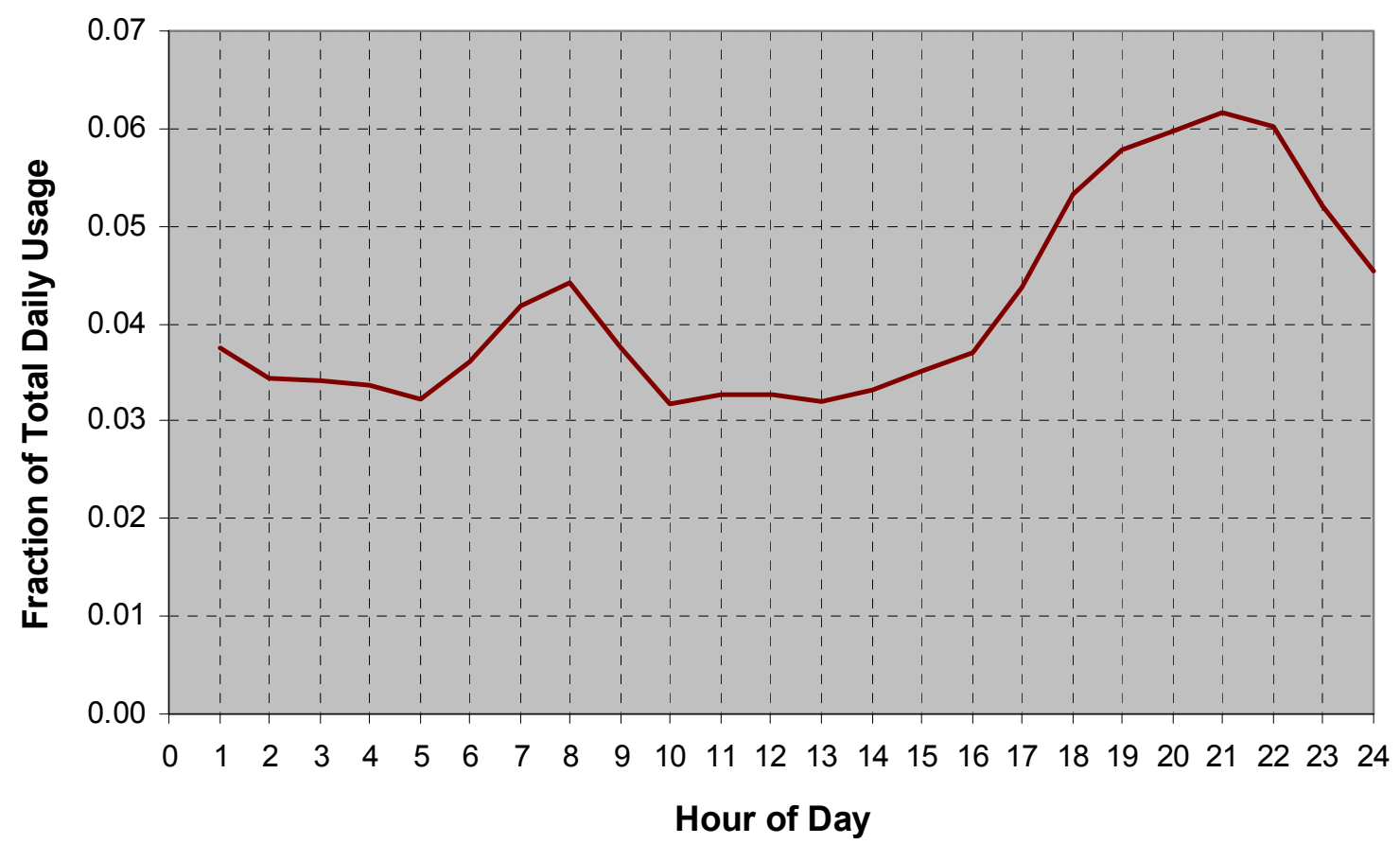

Figure 13. Miscellaneous electric load normalized energy-use profile 


\section{Site Generation}

If the Pre-Retrofit Case includes site electricity generation equipment, such as a fuel cell, photovoltaic system, or wind turbine, then the total energy production shall be calculated using a generally accepted engineering methodology.

\section{Modeling the Post-Retrofit Case}

The Post-Retrofit Case is modeled either as-designed or as-built, depending on the status of the project. All parameters for the Post-Retrofit model shall be based on final design specifications or measured data, with the following exceptions and clarifications:

- Any house characteristics that are unknown or not part of the package of energy efficiency improvements shall be the same as the Pre-Retrofit Case.

- The effective leakage area for the Post-Retrofit Case shall be calculated based on blowerdoor testing conducted in accordance with ASTM E779. If the whole-house simulation tool cannot calculate hourly infiltration based on effective leakage area, an annual average natural infiltration rate may be used based on the guidelines in ASHRAE Standard 119, Section 5, and ASHRAE Standard 136, Section 4. It is recommended that blower-door measurements be supplemented with tracer-gas testing when possible.

- In order to treat mechanical ventilation in a neutral manner, additional air exchange resulting from mechanical ventilation shall be assumed for the model of the Post-Retrofit Case if it does not meet the ventilation guidelines of ASHRAE Standard 62.2-2004 (ASHRAE 2004b) for existing homes based on natural infiltration plus any existing mechanical ventilation system. The same approach used for Pre-Retrofit Case shall be used to calculate "supplemental" mechanical ventilation (Equation 5) based on a simple continuous exhaust fan designed to raise the total ventilation rate to the minimum values specified in Equation 4.1a of ASHRAE Standard 62.2-2004, taking into account any infiltration credit allowed under Section 4.1.3 and the actual mechanical ventilation system (if any) present in the house. Mechanical ventilation shall be combined with natural infiltration in accordance with Section 4.4 of ASHRAE Standard 136 to determine an approximate combined effective air change rate. The additional fan energy use associated with supplemental mechanical ventilation for the Post-Retrofit Case shall be calculated by multiplying the supplemental ventilation rate by $3.942 \mathrm{kWh} / \mathrm{cfm}$ (Equation 6). This energy shall be added to the energy used by any ventilation fan present in the house.

- For cooling equipment, the energy efficiency ratio (EER) along with part-load performance characteristics should be used in the annual simulation whenever possible. SEER is less desirable for an annual simulation, but is often the only information that is publicly available about a cooling system. If the actual EER for the Post-Retrofit Case is not readily available, Equation 8 may be used to make an approximate conversion from SEER to EER (Wassmer 2003).

\section{Equation 8: $\operatorname{EER}=-0.02 \times \mathrm{SEER}^{2}+1.12 \times \mathrm{SEER}$}

- The installation of energy-saving appliances or other equipment may reduce hot water consumption for certain end uses, reduce the internal sensible and latent loads, or affect the hourly operating profiles. Energy-savings calculations for the Post-Retrofit Case must take 
these effects into account using operating conditions based on rules developed for DOE residential appliance standards (DOE 1999) and the actual performance characteristics of the appliances. The number of cycles per year specified in the appliance standard for clothes washers is adjusted according to the number of bedrooms and the clothes washer capacity, using Equation 9:

\section{Equation 9: Clothes washer cycles per year $=(392) \times\left(1 / 2+N_{b r} / 6\right) \times 12.5 \mathrm{lb} / \mathrm{W}_{\text {test }}$}

where:

$$
\begin{aligned}
\mathrm{W}_{\text {test }}= & \text { maximum clothes washer test load weight found in } 10 \text { CFR part } 430 \text {, } \\
& \text { Subpt B, Appendix J1, as a function of the washer capacity in } \mathrm{ft}^{3} .
\end{aligned}
$$

A dryer usage factor (DUF) is applied to the clothes washer cycles to determine the number of annual dryer cycles, using Equation 10:

\section{Equation 10: Clothes dryer cycles per year $=$ DUF $x$ Clothes washer cycles per year}

where:

$$
\mathrm{DUF}=0.84 \text {. }
$$

The dishwasher annual operating cycles are similarly calculated, using Equation 11:

Equation 11: Dishwasher cycles per year $=(215) \times\left(1 / 2+N_{b r} / 6\right)$.

The BA Analysis Spreadsheet posted on the Building America Web site automates these calculations and is strongly recommended for the analysis of water-consuming appliances. The spreadsheet includes tabs to help analysts calculate energy savings for efficient clothes washers, clothes dryers, and dishwashers. It calculates the split between hot water and machine energy based on the EnergyGuide label, estimates dryer energy savings for clothes washers that reduce remaining moisture content, adjusts energy use for the assumption that both hot water and cold water temperatures for the house are different from the test values $\left(140^{\circ} \mathrm{F}\right.$ and $\left.60^{\circ} \mathrm{F} / 50^{\circ} \mathrm{F}\right)$, and adjusts for the type of controls present (thermostatic control valves, boost heating, cold water only). Both annual average and monthly average hot water usage are calculated in the spreadsheet.

- Energy savings for a new range/oven may only be credited if an energy factor has been determined in accordance with the DOE test procedures for cooking appliances (DOE 1993). Annual energy consumption is then estimated as the product of the energy factor and the annual useful cooking energy output as defined in the same test procedure. If the energy factor is unknown for a new range/oven, then it shall be assumed that the Post-Retrofit energy use for cooking is the same as the Pre-Retrofit case.

- Modifications to the Pre-Retrofit lighting profile and operating hours because of occupancy sensors or other controls may be considered for the Post-Retrofit Case, but negative and/or positive effects on space conditioning load must also be calculated, assuming $100 \%$ of interior lighting energy contributes to the internal sensible load.

- Large end uses that are not part of typical houses (such as swimming pools, Jacuzzis, workshops, etc.) shall not be explicitly included in the models for either the Pre- or PostRetrofit Case. The efficiency of these end uses should be addressed in a separate analysis. 
- For the Post-Retrofit Case, all site electricity generation is credited regardless of energy source. Residential-scale photovoltaic systems, wind turbines, fuel cells, and microcogeneration systems are all potential sources of electricity generated on the site. An offset must be applied to this electricity credit equal to the amount of purchased energy used in the on-site generation process. The credit for site generation shall be tracked separately from the whole-house energy analysis and reported as a separate line in the summary tables (discussed later in this report).

\section{Operating Conditions}

For consistency within Building America, operating conditions and other assumptions for existing homes shall be the same as those documented in the Benchmark for new construction (www.eere.energy.gov/buildings/building_america/pa resources.html). The Benchmark guidelines will be updated occasionally (every 2 or 3 years), so the most recent release posted on the BA web site should be consulted before developing the existing homes model.

The following operating conditions and other assumptions, based on the 2005 Benchmark Update, shall apply to both the Pre- and Post-Retrofit Cases. They are intended to represent the behavior of a typical set of occupants, not the current occupants of the house, because Building America is interested in estimating long-term energy savings of improvements to existing homes. The operating conditions are the same for both existing homes and new construction and are based on the cumulative experience of the authors through their work on Building America, HERS, Codes and Standards, and other residential energy efficiency programs.

- The following standard thermostat set points shall be used:

Set point for cooling: $76^{\circ} \mathrm{F}$ with no setup period

Set point for heating: $71^{\circ} \mathrm{F}$ with no setback period.

Actual thermostat set points used by the occupants may be used for the purpose of costbenefit analysis, but not as part of the formal energy-savings analysis reported to Building America.

- The natural ventilation schedule shall be set to reflect windows being opened occasionally. In situations in which there is a cooling load, the outdoor temperature is below the indoor temperature, and the windows are not already open, then the probability of the windows being opened shall be set at a constant $50 \%$. The natural ventilation rate shall be $5 \mathrm{ACH}$ unless each living area and bedroom provides at least two openings on different orientations and the net area of openings exceeds $12 \%$ of the floor area of the house (cross-ventilation), in which case a natural ventilation rate of $7 \mathrm{ACH}$ shall be used. If there are local circumstances that would tend to discourage window operation (pollution, security, community standards, etc), then it is acceptable to use a lower probability than $50 \%$, as long as the same natural ventilation schedule is applied to both the Pre- and Post-Retrofit Cases.

- Interior shading multiplier $=0.7$ during the cooling season and 0.85 during the heating season and during swing seasons when both cooling and heating occur. Specific guidelines for defining seasons are presented later in this section.

- Internal loads from lighting, appliances, and other equipment were discussed in previous sections. These loads are not necessarily the same for the Pre- and Post-Retrofit Cases; 
therefore, they are not considered operating conditions for the purposes of Building America performance analysis.

- The occupancy schedule is defined with the same level of detail as other internal load profiles. For typical Building America houses, the number of occupants shall be estimated based on the number of bedrooms using Equation 12.

\section{Equation 12: Number of occupants $=0.5 \times \mathrm{N}_{\mathrm{br}}+1.5$}

where:

$$
\mathrm{N}_{\mathrm{br}}=\text { Number of bedrooms. }
$$

Sensible and latent gains from occupants shall be accounted for separately, and different loads shall be applied in different space types for multi-zone models (Table 19). The occupant heat gains are based on ASHRAE recommendations (ASHRAE 2005). The average hourly occupancy profile is shown in Figure 14, and an example set of detailed hourly occupancy curves is shown in Figure 15. Detailed occupancy profiles based on different day and room types are available in spreadsheet format on the Building America Web site (www.eere.energy.gov/buildings/building_america/pa resources.html). These profiles, which were developed by NREL, are based on the basic ASHRAE occupancy schedule combined with engineering judgment.

- The internal mass of furniture and contents shall be equal to $8 \mathrm{lbs} / \mathrm{ft}^{2}$ of conditioned floor space. For solar distribution purposes, lightweight furniture covering $40 \%$ of the floor area shall be assumed. The actual thermal mass present in the house is not used for Building America existing homes analysis.

Table 19. Peak Sensible and Latent Heat Gain from Occupants (ASHRAE 2005)

\begin{tabular}{|l|c|}
\hline \multicolumn{1}{|c|}{ Multiple Zones } & Internal Load (Btu/person/hr) \\
\hline Living Area Sensible Load & 230 \\
\hline Living Area Latent Load & 190 \\
\hline Bedroom Area Sensible Load & 210 \\
\hline Bedroom Area Latent Load & 140 \\
\hline \multicolumn{1}{|c|}{ Single Zone } & Internal Load (Btu/person/hr) \\
\hline Sensible Load & 220 \\
\hline Latent Load & 164 \\
\hline
\end{tabular}




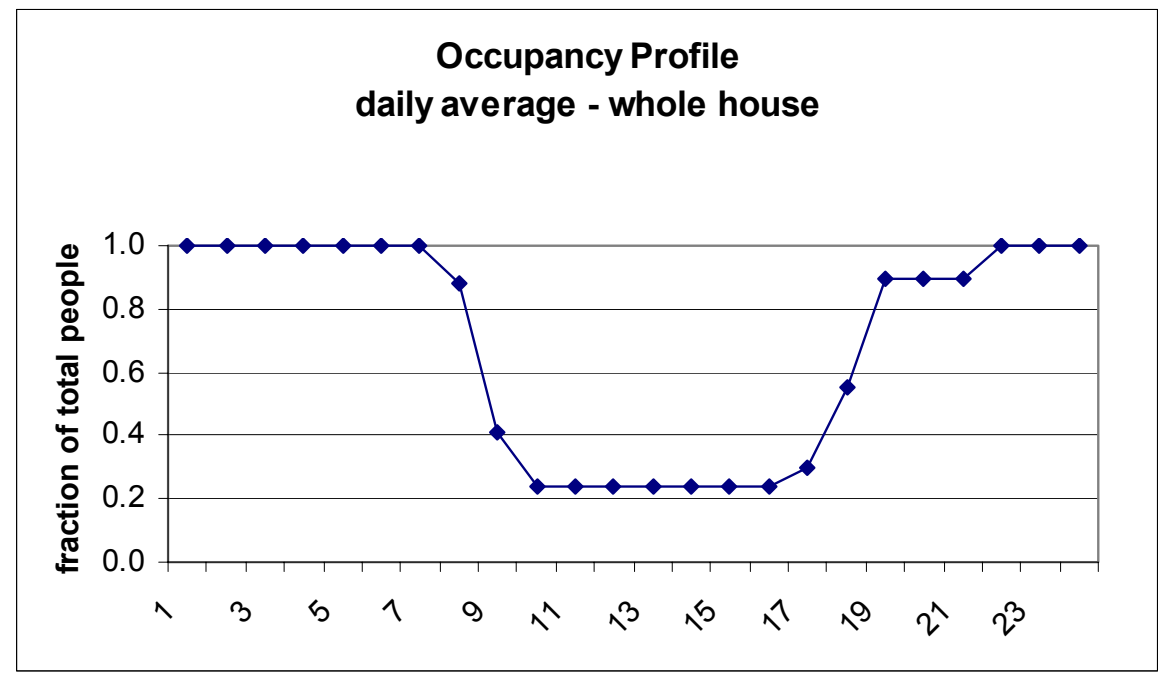

Figure 14. Average hourly load profile from occupants for all day-types and family types (16.5 hours/day/person total)

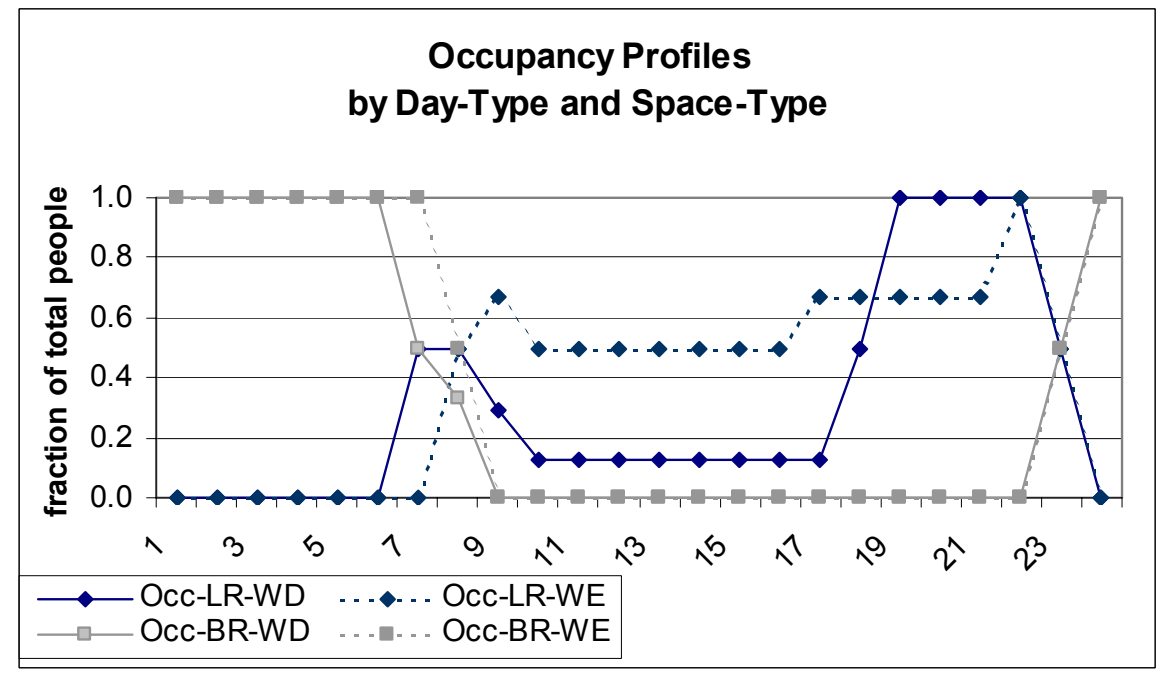

Figure 15. Detailed hourly load profiles resulting from occupants being in different parts of the house on weekdays (WD) and weekends (WE)

- Weather data shall be based on typical meteorological year (TMY2) data (NREL 1995) from 1961 through 1990 or equivalent data for the nearest weather station.

- Heating and cooling shall only occur during certain months of the year in accordance with the guidelines presented below. Alternate operating profiles may be acceptable with sufficient justification. The heating and cooling seasons shall be determined on the basis of the monthly average temperatures (MAT) and the 99\% (annual, not seasonal) winter design temperatures (WDT) based on TMY2 data or the 2005 ASHRAE Handbook of Fundamentals for the nearest location, in accordance with the following procedures: 


\section{Step 1. MAT Basis}

- (I) The heating system shall be enabled for a month in which the MAT is less than $71.5^{\circ} \mathrm{F}$.

- (II) The cooling system shall be enabled for a month in which the MAT is greater than $66^{\circ} \mathrm{F}$.

\section{Step 2. WDT and SDT}

- (I) The heating system shall be enabled in December and January if the WDT is less than or equal to $59^{\circ} \mathrm{F}$, regardless of the outcome in Step 1 above.

- (II) The cooling system shall be enabled in July and August regardless of the outcome in Step 1 above.

\section{Step 3. Swing Season Adjustment}

- (I) If, based on Steps 1 and 2 above, there are two consecutive months in which the heating system is enabled the first month and the cooling system is enabled the following month, or vice versa, then both the heating system and the cooling system shall be enabled for both of these months.

These heating and cooling season procedures are automated in the BA Analysis Spreadsheet (www.eere.energy.gov/buildings/building america/pa resources.html).

\section{Reporting Energy Use and Energy Savings}

Reporting energy use and energy savings in a consistent format is an important component of Building America analysis. The following tables shall be supplied with the analysis report for every existing homes project.

Table 20 shows an example of a site energy consumption report for a hypothetical project, before and after the retrofits are performed. Similar information based on source energy is presented in Table 21, along with percent energy savings for each end use. End uses are described in more detail in Table 22.

The "Percent of End Use" column in Table 21 shows the Post-Retrofit energy savings for each end use as a fraction of the energy use in the Pre-Retrofit Case. The "Percent of Total" columns show the contribution of each end use toward an overall energy-reduction goal.

Source energy is determined using Equation 13. The site to source multiplier for energy sources other than electricity or natural gas is assumed to be 1.0.

$$
\begin{aligned}
& \text { Equation 13: Source } \mathbf{M B t u}=\mathbf{k W h} \bullet \mathbf{3 . 4 1 2} \bullet \mathbf{M}_{\mathbf{e}} / \mathbf{1 0 0 0}+\text { therms } \bullet \mathbf{M}_{\mathbf{g}} / \mathbf{1 0} \\
& \text { where: } \\
& \qquad \begin{array}{l}
\mathrm{M}_{\mathrm{e}}=3.16=\text { site to source multiplier for electricity (DOE 2002) } \\
\mathrm{M}_{\mathrm{g}}=1.02=\text { site to source multiplier for natural gas (DOE 1995). }
\end{array}
\end{aligned}
$$


Table 20. Example Summary of Site Energy Consumption by End-use for an Existing Homes Project

\begin{tabular}{|c|c|c|c|c|}
\hline \multirow[b]{3}{*}{ End Use } & \multicolumn{4}{|c|}{ Annual Site Energy } \\
\hline & \multicolumn{2}{|c|}{ Pre-Retrofit } & \multicolumn{2}{|c|}{ Post-Retrofit } \\
\hline & (kWh) & (therms) & (kWh) & (therms) \\
\hline Space Heating & 11,225 & 0 & 4,397 & 0 \\
\hline Space Cooling & 2,732 & 0 & 902 & 0 \\
\hline $\mathrm{DHW}$ & 4,837 & 0 & 1,351 & 0 \\
\hline Lighting & 3,110 & & 1,204 & \\
\hline Appliances + MEL & 7,646 & 0 & 7,436 & 0 \\
\hline OA Ventilation & 400 & & 400 & \\
\hline Total Usage & 29,950 & 0 & 15,690 & 0 \\
\hline Site Generation & 0 & & 7,402 & \\
\hline Net Energy Use & 29,950 & 0 & 8,289 & 0 \\
\hline
\end{tabular}


Table 21. Example Summary of Source Energy Consumption by End-use for an Existing Homes Project

\begin{tabular}{|l|c|c|c|c|}
\cline { 2 - 5 } \multicolumn{1}{c|}{} & \multicolumn{2}{c|}{$\begin{array}{c}\text { Estimated Annual Source } \\
\text { Energy }\end{array}$} & \multicolumn{2}{c|}{ Source Energy Savings } \\
\cline { 2 - 5 } \multicolumn{1}{c|}{} & $\begin{array}{c}\text { Pre-Retrofit } \\
\text { (MBtu/yr) }\end{array}$ & $\begin{array}{c}\text { Post-Retrofit } \\
\text { (MBtu/yr) }\end{array}$ & $\begin{array}{c}\text { Percent of } \\
\text { End-Use }\end{array}$ & Percent of \\
End Use & 115 & 45 & $61 \%$ & $23 \%$ \\
\hline Space Heating & 28 & 9 & $67 \%$ & $6 \%$ \\
\hline Space Cooling & 50 & 14 & $72 \%$ & $12 \%$ \\
\hline DHW & 32 & 12 & $61 \%$ & $6 \%$ \\
\hline Lighting & 78 & 76 & $3 \%$ & $1 \%$ \\
\hline Appliances + MEL & 4 & 4 & $0 \%$ & $0 \%$ \\
\hline OA Ventilation & 307 & 161 & $48 \%$ & $48 \%$ \\
\hline Total Usage & 0 & -76 & $72 \%$ & $72 \%$ \\
\hline Site Generation & 307 & 85 & & $25 \%$ \\
\hline Net Energy Use & & & & \\
\hline
\end{tabular}


Table 22. End-Use Categories

\begin{tabular}{|l|l|l|}
\hline \multicolumn{1}{|c|}{ End Use } & \multicolumn{1}{|c|}{ Potential Electric Usage } & \multicolumn{1}{c|}{ Potential Gas Usage } \\
\hline $\begin{array}{l}\text { Space } \\
\text { Heating }\end{array}$ & $\begin{array}{l}\text { Supply fan during space heating, heat } \\
\text { pump, heat-pump supplemental heat, } \\
\text { water-boiler heating elements, water- } \\
\text { boiler circulation pump, electric- } \\
\text { resistance heating, heat-pump crankcase } \\
\text { heat, heating-system auxiliary }\end{array}$ & $\begin{array}{l}\text { Gas furnace, gas boiler, } \\
\text { gas back-up heat-pump } \\
\text { supplemental heat, gas } \\
\text { ignition stand-by }\end{array}$ \\
\hline $\begin{array}{l}\text { Space } \\
\text { Cooling }\end{array}$ & $\begin{array}{l}\text { Central split-system A/C, packaged A/C } \\
\text { (window or through-the-wall), supply-an } \\
\text { energy during space cooling, A/C } \\
\text { crankcase heat, cooling-system auxiliary }\end{array}$ & $\begin{array}{l}\text { Gas absorption chiller } \\
\text { (rare) }\end{array}$ \\
\hline DHW & $\begin{array}{l}\text { Electric hot water heater, heat-pump } \\
\text { water heater, hot water circulation pumps }\end{array}$ & Gas hot water heater \\
\hline Lighting & Indoor lighting, outdoor lighting & None \\
\hline $\begin{array}{l}\text { Appliances \& } \\
\text { MEL }\end{array}$ & $\begin{array}{l}\text { Refrigerator, electric clothes dryer, gas } \\
\text { clothes dryer (motor), cooking, } \\
\text { miscellaneous electric loads }\end{array}$ & $\begin{array}{l}\text { Cooking, gas clothes } \\
\text { dryer }\end{array}$ \\
\hline $\begin{array}{l}\text { OA } \\
\text { Ventilation }\end{array}$ & $\begin{array}{l}\text { Ventilation fans, supply-air fan during } \\
\text { ventilation mode }\end{array}$ & None \\
\hline $\begin{array}{l}\text { Site } \\
\text { Generation }\end{array}$ & \begin{tabular}{l} 
Photovoltaic electric generation \\
\hline
\end{tabular} & None \\
\hline
\end{tabular}

Table 23 reports energy savings for individual energy efficiency measures applied to the PreRetrofit Case, in terms of site energy, source energy, and energy cost. "Source Energy Savings $\%$ " is determined by comparing the source energy for each measure increment to the source energy for the Pre-Retrofit Case (i.e., the first row). In this column, the incremental savings for each measure are added to the savings for all the previous measures. The final row of the column is the overall energy savings achieved for the Post-Retrofit Case.

When available, actual energy tariffs for the house shall be used to determine whole-building energy costs. Peak hourly energy consumption should also be reported Pre- and Post-Retrofit for every project. Peak energy is based on the hour with the greatest gas or electric energy consumption during the course of 1 year, as determined by the hourly simulation. 
Table 23. Example Measure Savings Report for an Existing Homes Project ${ }^{2}$

\begin{tabular}{|c|c|c|c|c|c|c|c|c|c|c|}
\hline & & & & & \multicolumn{2}{|c|}{ National Average } & \multicolumn{4}{|c|}{ Economics (Local Costs) } \\
\hline & \multicolumn{2}{|c|}{ Site Energy } & \multicolumn{2}{|c|}{ Source Energy } & \multicolumn{2}{|c|}{ Energy Cost } & \multicolumn{2}{|c|}{ Energy Cost } & \multicolumn{2}{|c|}{ Measure Package } \\
\hline Increment & $(\mathrm{kWh})$ & (therms) & (MBtu) & $\begin{array}{l}\text { Savings } \\
(\%)\end{array}$ & $(\$ / y r)$ & $\begin{array}{c}\text { Savings } \\
(\%)\end{array}$ & $(\$ / y r)$ & $\begin{array}{c}\text { Savings } \\
(\%)\end{array}$ & $\begin{array}{l}\text { Value } \\
(\$ / y r)\end{array}$ & $\begin{array}{c}\text { Savings } \\
(\$ / y r)\end{array}$ \\
\hline Pre-Retrofit & 29,950 & 0 & 306.9 & & $\$ 2,995$ & & $\$ 2,950$ & & & \\
\hline + Improved walls & 27,779 & 0 & 284.6 & $7 \%$ & $\$ 2,778$ & $7 \%$ & $\$ 2,736$ & $7 \%$ & $\$ 190.4$ & $\$ 190$ \\
\hline ++ Low-E Windows & 25,810 & 0 & 264.5 & $14 \%$ & $\$ 2,581$ & $14 \%$ & $\$ 2,542$ & $13 \%$ & $\$ 193.9$ & $\$ 384$ \\
\hline ++ Smaller A/C ( $5 \geq 4$ tons) & 25,420 & 0 & 260.5 & $15 \%$ & $\$ 2,542$ & $15 \%$ & $\$ 2,504$ & $14 \%$ & $\$ 38.4$ & $\$ 423$ \\
\hline $\begin{array}{l}++ \text { Including Basement Wall } \\
\text { Insulation }\end{array}$ & 25,170 & 0 & 257.9 & $16 \%$ & $\$ 2,517$ & $16 \%$ & $\$ 2,479$ & $15 \%$ & $\$ 24.6$ & $\$ 447$ \\
\hline ++ Ground Source HP (+DHW) & 19,331 & 0 & 198.1 & $35 \%$ & $\$ 1,933$ & $35 \%$ & $\$ 1,904$ & $35 \%$ & $\$ 575.1$ & $\$ 1,023$ \\
\hline ++ Solar DHW & 17,718 & 0 & 181.5 & $41 \%$ & $\$ 1,772$ & $41 \%$ & $\$ 1,745$ & $40 \%$ & $\$ 158.9$ & $\$ 1,181$ \\
\hline ++ Lighting, Appliances and Plug & 15,690 & 0 & 160.8 & $48 \%$ & $\$ 1,569$ & $48 \%$ & $\$ 1,545$ & $47 \%$ & $\$ 199.8$ & $\$ 1,381$ \\
\hline ++ PV (Post-Retrofit) & 8,288 & 0 & 84.9 & $72 \%$ & $\$ 829$ & & $\$ 816$ & $72 \%$ & $\$ 729.0$ & $\$ 2,110$ \\
\hline
\end{tabular}

\section{Acknowledgments}

The author thanks the NREL staff for their ideas and inputs on this project, especially Ren Anderson, Paul Torcellini, Ali Jalalzedah, Mark Eastment, and Nancy Wells. The author also acknowledges the resources and guidance provided by Ed Pollock and George James of the U.S. Department of Energy Building America Program.

\footnotetext{
${ }^{2}$ Calculated using national average electric cost $=\$ 0.10 / \mathrm{kWh}$ and national average gas cost $=\$ 0.50 /$ therm.
} 


\section{References}

ASTM (American Society for Testing and Materials). 2003. Standard Test Method for Determining Air Leakage Rate by Fan Pressurization. ASTM E779-03. Conshohocken, PA: American Society for Testing and Materials.

ASTM (American Society for Testing and Materials). 1994. Standard Test Methods for Determining External Air Leakage of Air Distribution Systems by Fan Pressurization. ASTM E1554-94. Conshohocken, PA: American Society for Testing and Materials.

ASHRAE (American Society of Heating, Refrigerating, and Air-Conditioning Engineers). 2004a. HVAC Systems and Equipment Handbook. Atlanta, GA: American Society of Heating, Refrigerating, and Air-Conditioning Engineers.

ASHRAE (American Society of Heating, Refrigerating, and Air-Conditioning Engineers). 2004b. Ventilation and Acceptable Indoor Air Quality in Low-Rise Residential Buildings, ASHRAE Standard 62.2-2004, Atlanta, GA: American Society of Heating, Refrigerating, and Air-Conditioning Engineers

ASHRAE (American Society of Heating, Refrigerating, and Air-Conditioning Engineers). 2005. Fundamentals Handbook. Atlanta, GA: American Society of Heating, Refrigerating, and Air-Conditioning Engineers.

ASHRAE (American Society of Heating, Refrigerating, and Air-Conditioning Engineers). 1988. Air Leakage Performance for Detached Single-Family Residential Buildings, ASHRAE Standard 119-1988, Atlanta, GA: American Society of Heating, Refrigerating, and AirConditioning Engineers.

ASHRAE (American Society of Heating, Refrigerating, and Air-Conditioning Engineers). 1993. A Method of Determining Air Change Rates in Detached Dwellings, ASHRAE Standard 136-1993, Atlanta, GA: American Society of Heating, Refrigerating, and AirConditioning Engineers.

ASHRAE (American Society of Heating, Refrigerating, and Air-Conditioning Engineers). 2001. Method of Test for Determining the Design and Seasonal Efficiencies of Residential Thermal Distribution Systems, Proposed ASHRAE Standard 152P, Atlanta, GA: American Society of Heating, Refrigerating, and Air-Conditioning Engineers.

Burch, J., and Erickson, P. 2004. "Using Ratings Data to Derive Simulation-Model Inputs for Storage-Tank Water Heaters." 2004 National Solar Energy Conference. Boulder, CO: American Solar Energy Society.

DOE (U.S. Department of Energy). July 1993. Technical Support Document for Residential Cooking Products. Volume 2. Available online at www.eere.energy.gov/buildings/appliance standards/residential/cooking_products 0998 r.html (accessed July 2005). Washington, D.C.: U.S. Department of Energy.

DOE (U.S. Department of Energy). December 1995. Measuring Energy Efficiency in the United States Economy: A Beginning. Chapter 7. Available online at www.eia.doe.gov/emeu/efficiency/ee report html.htm (accessed May 2004). Washington, D.C.: U.S. Department of Energy. 
DOE (U.S. Department of Energy). 1999. Code of Federal Regulations Title 10, Energy, Part 430, Subpart B, Appendcies C, D, J and J1, Washington, D.C.: U.S. Department of Energy.

DOE (U.S. Department of Energy). 2000a. Technical Support Document: Energy Efficiency Standards for Consumer Products: Residential Water Heaters. Appendix D-2. Available online at www.eere.energy.gov/buildings/appliance standards/residential/waterheat 0300 r.html (accessed May 2004). Washington, D.C.: U.S. Department of Energy.

DOE (U.S. Department of Energy). 2000b. Final Rule Technical Support Document: Energy Efficiency Standards for Consumer Products: Clothes Washers. Available online at www.eere.energy.gov/buildings/appliance standards/residential/clwash $0900 \mathrm{r.html}$ (accessed July 2005). Washington, D.C.: U.S. Department of Energy.

DOE (U.S. Department of Energy). 2001. Residential Energy Consumption Survey. Washington, DC: U.S. Department of Energy.

DOE (U.S. Department of Energy). 2002. Technical Support Document: Energy Efficiency Standards for Consumer Products: Residential Central Air Conditioners and Heat Pumps. Available online at www.eere.energy.gov/buildings/appliance standards/residential/central ac hp.html (accessed August 2005). Washington, D.C.: U.S. Department of Energy.

DOE (U.S. Department of Energy). 2003. New and Alternative Insulation Materials and Products. Energy Savers Fact Sheet. Available online at www.eere.energy.gov/consumerinfo/factsheets/eb9.html (accessed August 2005). Washington, D.C.: U.S. Department of Energy.

DOE (U.S. Department of Energy). 2004a. Technical Support Document: Energy Efficiency Program for Consumer Products: Energy Conservation Standards for Residential Furnaces and Boilers. Available online at www.eere.energy.gov/buildings/appliance standards/residential/furnaces boilers $1113 \mathrm{r}$ .html (accessed August 2005). Washington, D.C.: U.S. Department of Energy.

DOE (U.S. Department of Energy). 2004b. 2004 Buildings Energy Databook. Available online at http://btscoredatabook.eren.doe.gov/ (accessed August 2005). Washington, D.C.: U.S. Department of Energy.

EPRI (Electric Power Research Institute). 1986. Trends in the Energy Efficiency of Residential Electric Appliances. EM-4539, Research Project 2034-9. Palo Alto, CA: Electric Power Research Institute.

EPRI (Electric Power Research Institute). 1987. TAG Technical Assessment Guide: Volume 2: Electricity End Use. EPRI P-4463-SR. Palo Alto, CA: Electric Power Research Institute.

E-Source. 1993. Space Heating Technology Atlas. Boulder, CO: E-Source Inc.

E-Star Colorado. 2005. R-Value Table. Available at www.e-star.com/ecalcs/table_rvalues.html (accessed August 2005). 
Hancock, E., Norton, P., and Hendron, R. 2002. Building America System Performance Test Practices: Part 2, Air-Exchange Measurements. NREL/TP-550-30270. Golden, CO: National Renewable Energy Laboratory.

Hendron, R. 2005. Building America Research Benchmark Definition, Updated December 29, 2004. NREL/TP-550-37529. Golden, CO: National Renewable Energy Laboratory.

Hendron, R., and Eastment, M. 2006. "Development of an Energy-Savings Calculation Methodology for Residential Miscellaneous Electric Loads." ACEEE Summer Study on Energy Efficiency in Buildings. Washington, D.C.: American Council for an EnergyEfficient Economy (ACEEE).

Huang, J.; and Gu, L. 2002. Prototype Residential Buildings to Represent the U.S. Housing Stock. Draft LBNL Report. Berkeley, CA: Lawrence Berkeley National Laboratory.

Judkoff, R.; Balcomb, J. D.; Hancock, C. E.; Barker, G.; and Subbarao, K. 2000. Side-By-Side Thermal Tests of Modular Offices: A Validation Study of the STEM Method. NREL Technical Report TP-550-23940. Golden, CO: National Renewable Energy Laboratory.

Matson, N., Wray, C., Walker, I., and Sherman, M. 2002. Potential Benefits of Commissioning California Homes. LBNL-48258. Berkeley, CA: Lawrence Berkeley National Laboratory.

National Renewable Energy Laboratory (NREL). 1995. Solar Radiation Data Manual for Buildings. NREL/TP-463-7904. Golden, CO: NREL.

Parker, D., McIlvaine, J., Barkaszi, S., Beal, D., and Anello, M. 2000. Laboratory Testing of the Reflectance Properties of Roofing Material. FSEC-CR670-00. Cocoa, FL: Florida Solar Energy Center.

Pratt, R., Conner, C., Richman, E., Ritland, K., Sandusky, W., and Taylor, M. 1989. Description of Electric Energy Use in Single-Family Residences in the Pacific Northwest-End-Use Load and Consumer Assessment Program (ELCAP), DOE/BP-13795-21. Richland, WA: Pacific Northwest National Laboratory.

Residential Energy Services Network (RESNET). 2002. Mortgage Industry National Home Energy Rating Systems Accreditation Standards. Chapter 3, pp. 29-54. San Diego, CA: Residential Energy Services Network.

Szydlowski, R., and Cleary, P. 1988. "In-Situ Appliance Efficiency Audit Procedures." ASHRAE Transactions, v.94, pt.1, p.1107-1023.

Wassmer, M. 2003. A Component-Based Model for Residential Air Conditioner and Heat Pump Energy Calculations. Masters Thesis, University of Colorado at Boulder.

Wenzel, T., Kooney, J., Rosenquist, G., Sanchez, M., and Hanford, J. 1997. Energy Data Sourcebook for the U.S. Residential Sector. LBL-40297. Berkeley, CA: Lawrence Berkeley National Laboratory. 


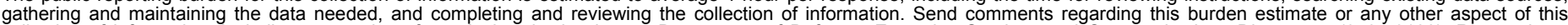

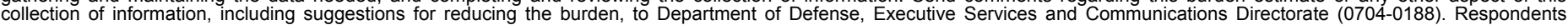

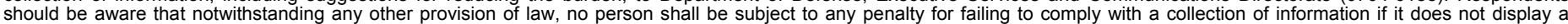

currently alid OMB control number.

PLEASE DO NOT RETURN YOUR FORM TO THE ABOVE ORGANIZATION.

\begin{tabular}{ll|l}
\hline 1. & $\begin{array}{l}\text { REPORT DATE }(D D-M M-Y Y Y Y) \\
\text { May } 2006\end{array}$ & $\begin{array}{l}\text { 2. } \\
\text { REPORT TYPE } \\
\text { Technical Report }\end{array}$ \\
4. & $\begin{array}{l}\text { TITLE AND SUBTITLE } \\
\text { Building America Performance Analysis Procedures for Existing } \\
\text { Homes }\end{array}$
\end{tabular}

Homes

3. DATES COVERED (From - To)

5a. CONTRACT NUMBER

DE-AC36-99-G010337

5b. GRANT NUMBER

5c. PROGRAM ELEMENT NUMBER

5d. PROJECT NUMBER

NREL/TP-550-38238

5e. TASK NUMBER

BET6.8004

5f. WORK UNIT NUMBER
7. PERFORMING ORGANIZATION NAME(S) AND ADDRESS(ES)

National Renewable Energy Laboratory

1617 Cole Blvd.

Golden, CO 80401-3393

9. SPONSORING/MONITORING AGENCY NAME(S) AND ADDRESS(ES)
8. PERFORMING ORGANIZATION REPORT NUMBER

NREL/TP-550-38238

10. SPONSOR/MONITOR'S ACRONYM(S) NREL

11. SPONSORING/MONITORING AGENCY REPORT NUMBER

12. DISTRIBUTION AVAILABILITY STATEMENT

National Technical Information Service

U.S. Department of Commerce

5285 Port Royal Road

Springfield, VA 22161

13. SUPPLEMENTARY NOTES

14. ABSTRACT (Maximum 200 Words)

With more than 101 million residential households in the United States today, existing residential buildings represent a large potential energy savings. Thousands of these homes are renovated each year, Building America is investigating the ways to make existing homes more energy-efficient, based on lessons learned from research in new homes. The Building America program is aiming for a $20 \%-30 \%$ reduction in energy use in existing homes by 2020. The strategy is to establish technology pathways that reduce energy consumption cost-effectively. The existing buildings project focuses on finding ways to adapt the results from the new homes research to retrofit applications in existing homes. Research activities include a combination of computer modeling, field demonstrations, and long-term monitoring to support the development of integrated approaches to reduce energy use. Analytical tools are being developed to guide designers and builders in selecting the best approaches. DOE partners with the EPA to increase energy efficiency in existing homes through the Home Performance with ENERGY STAR® program.

15. SUBJECT TERMS

Building America; existing homes; performance analysis procedures; residential buildings; energy efficiency

\begin{tabular}{|c|c|c|c|c|}
\hline 16. SECURITY & CLASSIFICATI & N OF: & 17. LIMITATION & 18. NUMBER \\
\hline $\begin{array}{l}\text { a. REPORT } \\
\text { Unclassified }\end{array}$ & $\begin{array}{l}\text { b. ABSTRACT } \\
\text { Unclassified }\end{array}$ & $\begin{array}{l}\text { c. THIS PAGE } \\
\text { Unclassified }\end{array}$ & UL & \\
\hline
\end{tabular}

\begin{tabular}{l} 
19a. NAME OF RESPONSIBLE PERSON \\
19b. TELEPHONE NUMBER (Include area code) \\
\hline
\end{tabular}




\section{A Strong Energy Portfolio for a Strong America}

Energy efficiency and clean, renewable energy will mean a stronger economy, a cleaner environment, and greater energy independence for America. Working with a wide array of state, community, industry, and university partners, the U.S. Department of Energy's Office of Energy Efficiency and Renewable Energy invests in a diverse portfolio of energy technologies.

\section{Research and Development of Buildings}

Our nation's buildings consume more energy than any other sector of the U.S. economy, including transportation and industry. Fortunately, the opportunities to reduce building energy useand the associated environmental impacts-are significant.

DOE's Building Technologies Program works to improve the energy efficiency of our nation's buildings through innovative new technologies and better building practices. The program focuses on two key areas:

\section{- Emerging Technologies} Research and development of the next generation of energy-efficient components, materials, and equipment

- Technology Integration Integration of new technologies with innovative building methods to optimize building performance and savings

For more information contact: EERE Information Center 1-877-EERE-INF (1-877-337-3463) www.eere.energy.gov

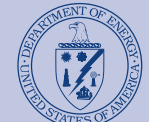

U.S. Department of Energy Energy Efficiency and Renewable Energy

An electronic copy of this publication is available on the Building America Web site at www.buildingamerica.gov

\section{Visit our Web sites at:}

\section{www.buildingamerica.gov}

www.pathnet.org

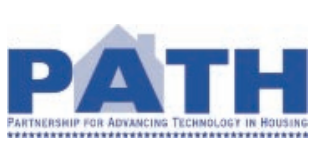

www.energystar.gov

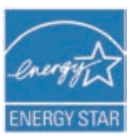
U.S. Department of Energy
Research Toward Zero Energy Homes

Building America Program

George S. James • New Construction • 202-586-9472• fax: 202-586-8134• e-mail: George.James@ee.doe.gov Terry Logee • Existing Homes • 202-586-1689 • fax: 202-586-4617• e-mail: terry.logee@ee.doe.gov Lew Pratsch • Integrated Onsite Power • 202-586-1512 • fax: 202-586-8185• e-mail: Lew.Pratsch@hq.doe.gov Building America Program • Office of Building Technologies, EE-2J • U.S. Department of Energy • 1000 Independence Avenue, S.W. • Washington, D.C. 20585-0121• www.buildingamerica.gov

Building Industry Research Alliance (BIRA)

Robert Hammon • ConSol • 7407 Tam 0'Shanter Drive \#200 • Stockton, CA 95210-3370 • 209-473-5000 • fax: 209-474-0817• e-mail: Rob@consol.ws• www.bira.ws

Building Science Consortium (BSC)

Betsy Pettit • Building Science Consortium (BSC) • 70 Main Street • Westford, MA $01886 \bullet 978-589-5100$ • fax: 978-589-5103 • e-mail: Betsy@buildingscience.com • www.buildingscience.com

Consortium for Advanced Residential Buildings (CARB)

Steven Winter • Steven Winter Associates, Inc. • 50 Washington Street • Norwalk, CT $06854 \bullet 203-857-0200 \bullet$ fax: 203-852-0741 • e-mail: swinter@swinter.com • www.carb-swa.com

\section{Davis Energy Group}

David Springer • Davis Energy Group $\bullet 123$ C Street • Davis, CA 95616 • 530-753-1100 • fax: 530-753-4125 • e-mail: springer@davisenergy.com • deg@davisenergy.com・ www.davisenergy.com/index.html

\section{IBACOS Consortium}

Brad Oberg •IBACOS Consortium • 2214 Liberty Avenue • Pittsburgh, PA $15222 \bullet 412-765-3664 \bullet$ fax: 412-765-3738 • e-mail: boberg@ibacos.com • www.ibacos.com

Industrialized Housing Partnership (IHP)

Subrato Chandra • Florida Solar Energy Center • 1679 Clearlake Road • Cocoa, FL 32922 • 321-638-1412 • fax: 321-638-1439• e-mail: subrato@fsec.ucf.edu • www.baihp.org

National Association of Home Builders (NAHB) Research Center

Tom Kenney • National Association of Home Builders (NAHB) Research Center • 400 Prince George's Boulevard • Upper Marlboro, MD 20774 • 301-430-6246• fax: 301-430-6180 • toll-free: 800-638-8556• www.nahbrc.org/

\section{National Renewable Energy Laboratory}

Ren Anderson • 1617 Cole Boulevard, MS-2722 • Golden, C0 80401 • 303-384-7433 • fax: 303-384-7540 •

e-mail: ren_anderson@nrel.gov • www.nrel.gov

Tim Merrigan • 1617 Cole Boulevard, MS-2722 • Golden, C0 80401 • 303-384-7349 • fax: 303-384-7540 • e-mail: tim_merrigan@nrel.gov • www.nrel.gov

\section{Oak Ridge National Laboratory}

Pat M. Love • P.0. Box $2008 \bullet$ One Bethel Valley Road • 0ak Ridge, TN 37831 • 865-574-4346 • fax: 865-574-9331 • e-mail: lovepm@ornl.gov • www.ornl.gov

Produced for the U.S. Department of Energy (DOE) by the National Renewable Energy Laboratory, a DOE national laboratory. May $2006 \bullet$ NREL/TP-550-38238

Printed with a renewable-source ink on paper containing at least $50 \%$ wastepaper, including $10 \%$ postconsumer waste. 